Recibido: 28 de agosto del 2016

Aprobado: 29 de setiembre del 2016

\title{
Sistema Web PARA LA ADMINISTRACIÓN DE ATENCIONES MÉDICAS Y MONITOREO DE LA UNIDAD DE TRIAJE en el hospital nacional Cayetano Heredia
}

\author{
Jennifer Duarte Jauler \\ Jennifer.duarte.jauler@gmail.com \\ Dubai World Port Callao. Callao, Perú
}

\section{Resumen}

El área de Emergencia del hospital nacional Cayetano Heredia no dispone de una infraestructura adecuada; a pesar de ello, la cantidad de pacientes ha crecido en los últimos años: La unidad de Triaje es una de las subáreas de Emergencia, cuya finalidad es clasificar a los pacientes de acuerdo a su nivel de prioridad a través de un diagnóstico rápido, para ofrecer un nivel de servicio adecuado a los pacientes críticos y optimizar los recursos hospitalarios. El presente artículo muestra el desarrollo de un sistema web para la integración de Triaje con las diferentes subáreas que comprenden el área de Emergencia, el cual permitiría brindar información consolidada y compartida en tiempo real, agilizando de esa manera el proceso de atención y una gestión adecuada de los recursos necesarios para los pacientes en estado crítico.

Palabras clave: triaje / atención en emergencias / medicina / tecnología

\section{Abstract}

Despite the lack of an appropriate infrastructure, the number of patients in the Emergency Area of Cayetano Heredia National Hospital has grown in the past few years. The Triage Area is a subarea of the Emergency Area, whose purpose is to classify patients according to their priority level through a quick diagnosis, in order to provide an appropriate level of service to critical patients and optimize the use of hospital resources. This article shows the development of a web system for the integration of the Triage Area with the other subareas of the Emergency area, which would allow the provision of consolidated and shared information in real time, thus speeding up attention process and improving the allocation of the resources critical patients need.

Keywords: triage / emergency attention / medicine / technology 


\section{Introducción}

\subsection{Antecedentes}

Simmons (2004) aborda al triaje como una técnica para priorizar atenciones de acuerdo al tratamiento que se tenga que efectuar al paciente. Nos muestra un sistema de triaje de trauma que clasifica cuatro categorías dentro de este criterio: fisiología, anatomía de la lesión, mecanismo de lesión y factores comórbidos.

Padmanabhan et al. (2006) describen la creación de un software denominado "iTriage", que ayuda en la toma de decisiones y significó una innovación al ser implementado en un PDA (Personal Digital Assistant), equipo muy utilizado a inicios del presente milenio. El mencionado software estaba enfocado en una metodología que indicaba los problemas a evitar al momento de generar una solución, lo que permitía realizar una mejor selección de la categoría a la que debería pertenecer el paciente de acuerdo a su necesidad, e identificar el atributo. Así se ratificaba un nivel de confianza mayor y se comprobaba la utilidad del software. De este modo, se concluyó que esta herramienta servía para evaluar qué tan adecuado era el sistema de clasificación de triaje en base a sus resultados, que podrían ser: a) los esperados o correctos, cuando el área de Triaje deriva al paciente correcto para atención en urgencia, b) sobretriaje, cuando se deriva innecesariamente un paciente para atención en urgencia, y c) subtriaje, cuando no se deriva a urgencia un paciente que así lo requiere. La herramienta impactó positivamente en la toma de decisiones, mediante medidas subjetivas y objetivas.

Sakanushi et al. (2011) abordan un sistema de triaje electrónico que reemplaza al triaje que se hacía anteriormente en papel, el cual consistía en diferenciar a un paciente internado a causa de un accidente, de acuerdo a una ficha que contaba con cuatro colores diferentes de etiqueta, según la prioridad a asignarse al paciente. Sin embargo, se presentaban muchos problemas debido a que a veces la etiqueta no mostraba la prioridad correcta, no se visualizaban las condiciones del paciente en el momento, y el oficial de triaje debía tomar su decisión en menos de un minuto. Sakanushi et al. proponen un software compuesto de etiquetas electrónicas y un servidor electrónico de triaje que miden signos vitales, se utiliza como herramienta para priorizar a los pacientes que lo necesitan. Además se tiene que el triaje electrónico puede diferenciar alguna anomalía que pueda tener la condición física del paciente, permitiendo la modificación de prioridades.

Yeh, Hwang, Huang, y Liu (2011) explican un software llamado U-Healthcare System diseñado para reforzar la calidad de atención de un paciente frecuente o crónico, el cual requiere para su uso que el paciente envíe signos vitales que apoyen al monitoreo a largo plazo; de esa manera, el médico puede analizarlos en el instante que se necesite para proveer una propuesta de tratamiento. 
Mizumoto, Imazu, Sun, Shibata, y Yasumoto (2012), mencionan los problemas del triaje simple mostrando el denominado criterio de evaluación START (Simple Triage and Rapid Treatment) para etiquetar al paciente con un color acorde a la gravedad:

- Etiqueta verde. Paciente puede caminar solo.

- Etiqueta amarilla. Se debe constatar que el paciente no puede caminar; puede respirar; presenta entre 10 y 30 respiraciones por minuto; y muestra un estado mental consciente (contesta preguntas y sigue indicaciones).

- Etiqueta roja. Paciente presenta dificultad al respirar. En caso la respiración esté normal, se mide el pulso y, dependiendo de ello, se conserva o no la etiqueta roja.

Mizumoto et al. (2012) sugieren el "eTriage" que se divide en tipo liviano y completo.

Ma, W., Goldstein, Ma, Y., Sun, y Burgess (2012) describen un website conocido como Medline, que sirve como medio de información para conocer temas acerca de la salud. Este nuevo sistema que Medline ha implementado mediante un aplicativo móvil tiene como objetivo captar clientes que utilizan teléfonos móviles y que podrían estar interesados en tener esta información cuando lo deseen.

Arias y Fisher (2013) señalan cómo se puede activar un centro de operaciones de emergencia (EOC, por sus siglas en inglés: Emergency Operation Center) cuando un gran evento supone un riesgo potencial para un significativo número de personas. Este sistema de gestión de emergencias requiere la captura de conocimiento de los usuarios de destino, por lo que está mejor provisto, con la aplicación de metodologías cualitativas para trabajo de campo y entrevistas a usuarios. Arias y Fischer (2013) propusieron una metodología para minimizar las interrupciones en el flujo de trabajo de los profesionales de la gestión de emergencias, sin comprometer el valor de la recogida de datos.

De los trabajos revisados se aprecia la utilidad de los sistemas de información, los cuales brindan beneficios a los pacientes para asegurar su integridad; sin embargo, estos sistemas suelen trabajar en forma independiente y no se ajustan a la realidad de los hospitales públicos peruanos. En la tabla 1 se presenta un cuadro de los sistemas propuestos en comparación con la solución planteada en la presente investigación (SIGEST): 
Tabla 1. Comparación de las soluciones analizadas

\begin{tabular}{|c|c|c|c|c|c|c|}
\hline \multirow[b]{2}{*}{ Criterio } & \multicolumn{6}{|c|}{ Producto } \\
\hline & iTriage & Triaje electrónico & $\begin{array}{l}\text { U- Healthcare- } \\
\text { System }\end{array}$ & eTriage & MedLine & SIGEST \\
\hline Tecnología & Java & RFID & Java & - & Java & $\begin{array}{c}\text { ASP.NET } \\
\text { Web }\end{array}$ \\
\hline $\begin{array}{l}\text { Priorización de } \\
\text { pacientes }\end{array}$ & Sí & Sí & Sí & Sí & No & Sí \\
\hline $\begin{array}{l}\text { Administración de } \\
\text { usuario y perfiles }\end{array}$ & No & No & No & No & No & Sí \\
\hline $\begin{array}{l}\text { Reportes de } \\
\text { atención por turno }\end{array}$ & No & No & No & No & No & Sí \\
\hline $\begin{array}{l}\text { Reportes } \\
\text { estadísticos con } \\
\text { indicadores }\end{array}$ & No & No & No & No & No & Sí \\
\hline $\begin{array}{l}\text { Registro de } \\
\text { atenciones }\end{array}$ & Sí & Sí & Sí & No & No & Sí \\
\hline $\begin{array}{l}\text { Reporte de } \\
\text { historiales } \\
\text { de atenciones }\end{array}$ & No & No & No & No & No & Sí \\
\hline $\begin{array}{l}\text { Administración de } \\
\text { pacientes atendi- } \\
\text { dos }\end{array}$ & Sí & No & Sí & No & No & Sí \\
\hline $\begin{array}{l}\text { Búsqueda } \\
\text { y registro } \\
\text { de atenciones } \\
\text { por tópicos }\end{array}$ & No & No & No & No & No & Sí \\
\hline $\begin{array}{l}\text { Integración con } \\
\text { otras unidades u } \\
\text { áreas }\end{array}$ & No & No & No & No & No & Sí \\
\hline
\end{tabular}

Elaboración propia

En la tabla 1 se muestra que SIGEST permite integrar, almacenar y consultar la información en tiempo real, haciendo de ella una solución con ventajas competitivas.

\subsection{Formulación del problema}

El hospital nacional Cayetano Heredia cuenta con una gran demanda anual de pacientes en el área de Emergencia (ver figura 1); en el año 2014 se atendieron aproximadamente 250 
pacientes al día. La mencionada área se caracteriza por brindar una atención inmediata de acuerdo a la enfermedad o patología que pone en riesgo la vida del paciente.

Figura 1. Atenciones por año en el área de Emergencia

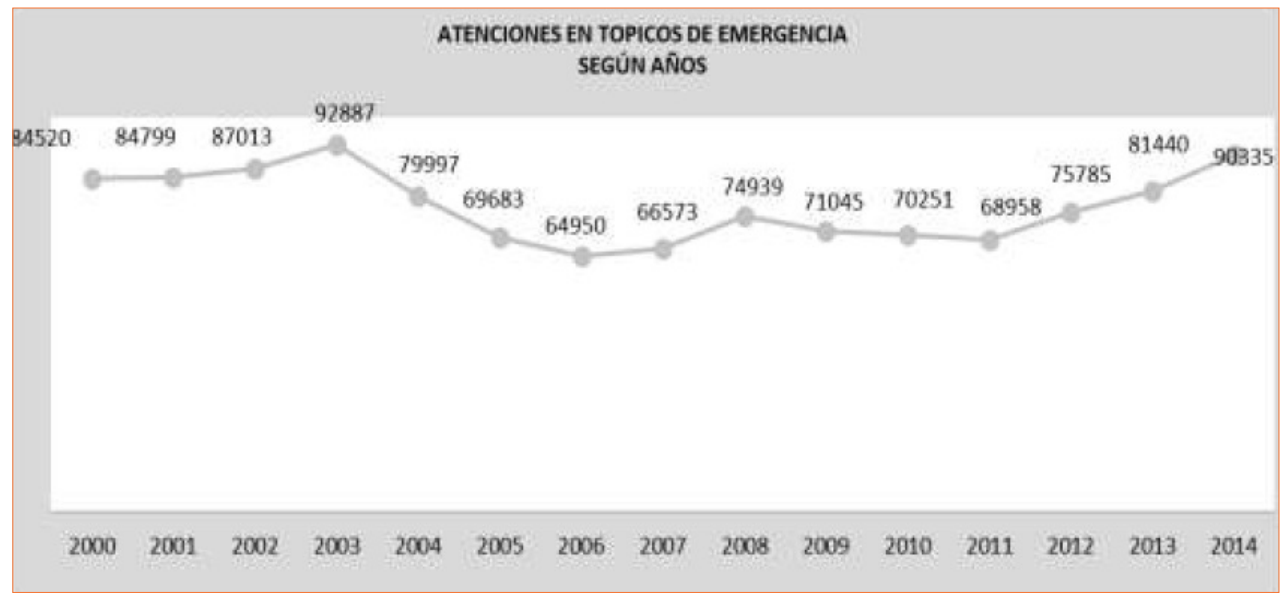

Fuente: Oficina de estadística e informática del hospital Cayetano Heredia

De acuerdo al artículo 3 del título II definiciones del Reglamento de la Ley Nº 27604 (2002), "se entiende por emergencia médica a toda condición repentina e inesperada que requiere atención inmediata, al poner en peligro inminente la vida, la salud o que puede dejar secuelas invalidantes en el paciente".

Sin embargo, los pacientes que ingresan por el área de Emergencia suelen pensar que cualquier complicación es una urgencia, ya sea por su poca disponibilidad de tiempo o porque el proceso de atención ambulatoria es engorroso, o porque toma demasiado tiempo acceder al servicio. Esto trae como consecuencia que la citada área del hospital no se abastezca para atenderlos.

A continuación se muestran los principales problemas (ver figura 2) que se presentan en los establecimientos del Ministerio de Salud del Perú (Minsa) - entidad a la cual pertenece el Hospital Cayetano Heredia - , donde se destaca el elevado tiempo de espera en el proceso de atención en Emergencias. 
Figura 2. Problemas presentados en los establecimientos de salud del Minsa

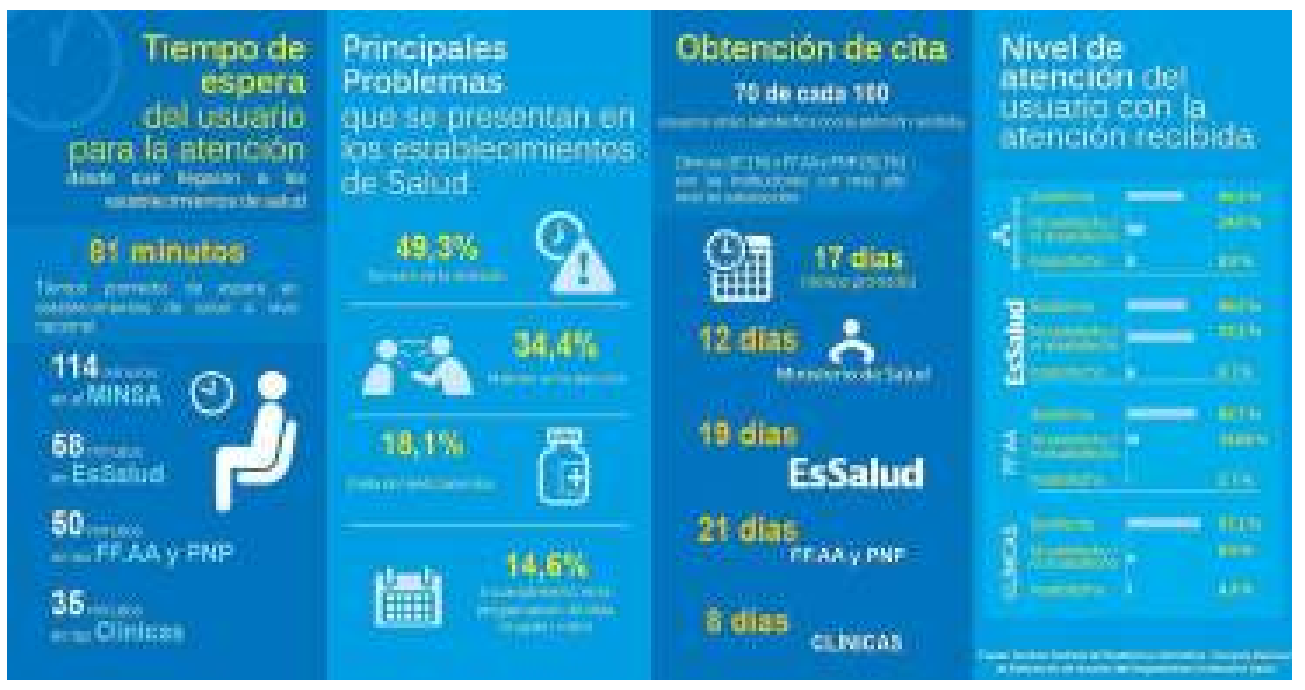

Fuente: INEI. Encuesta nacional de satisfacción de usuarios del aseguramiento universal de salud

Como ya se ha mencionado, en el área de Emergencia se encuentra la subárea de Triaje, que es la encargada de realizar una evaluación rápida sobre el tipo de enfermedad o patología que presente el paciente y luego derivarlo, de acuerdo al resultado, al tópico o a consultorios según uno de los cuatro niveles de prioridad:

El nivel 1 es el de extrema urgencia, donde los pacientes son enviados directamente a shock trauma, los de nivel 2 son derivados a tópicos de medicina, ginecología y cirugía; y los de niveles 3 y 4 son asignados a consultoría interna, también llamada consultoría de atención inmediata (Ministerio de Salud, 2006).

No obstante, pese a la rapidez que pueda tener el médico encargado en el registro de los datos del paciente y la derivación correspondiente, existen tiempos de inactividad holgados, y se carece de un nivel de integración necesario entre las subáreas ubicadas dentro del área de Emergencias, la cual no cuenta con un protocolo de comunicación adecuado, ni una estandarización de los datos básicos del paciente (nombre, edad, distrito, si cuenta con seguro, si vino acompañado, entre otros). Otro problema presentado en el área de Emergencia, y en especial en la subárea de Triaje, es que no se dispone de información histórica o de seguimiento de los pacientes, por lo que no hay manera de que la subárea de Triaje y las unidades asociadas (tópicos y consultoría), tengan conocimiento del estado del paciente durante el proceso de atención o de la culminación de dicho proceso. El desorden existente no permite brindar una atención adecuada a los casos de verdadera urgencia, lo cual se resume en lo siguiente: 
- Registro reiterativo de los datos del paciente, desde su ingreso y durante su paso por diferentes subáreas de Emergencia o unidades del hospital público.

- Falta de información consolidada en las diferentes subáreas de Emergencia, lo que complica tanto la labor del área de Emergencia como la del hospital.

- Desconocimiento por parte del personal del historial clínico del paciente, razón por la cual el tratamiento dado puede no ser el adecuado.

\subsection{Objetivos}

\subsubsection{Objetivo general}

Automatizar, mediante un sistema de información web, el proceso de atención médica en el área de Triaje y en el área de Emergencia de un hospital público para que el personal involucrado pueda consultar información consolidada e integrada con el historial clínico, así como agilizar las operaciones realizadas en el mismo.

\subsubsection{Objetivos específicos}

i. Analizar y obtener un diagnóstico del proceso de atención de los pacientes en la subárea de Triaje y en el área de Emergencia de un hospital público.

ii. Elaborar un plan de mejora del proceso de atención de los pacientes en la subárea de Triaje y en el área de Emergencia de un hospital público, que será implementado a través de un sistema web.

iii. Desarrollar el sistema web que permita gestionar todo el proceso de las atenciones médicas ofrecidas, desde el ingreso y clasificación del paciente en la subárea de Triaje.

iv. Rediseñar los registros de las atenciones que alimenten y centralicen la información de la historia clínica del paciente en un único documento virtual, para que puedan ser consultados en tiempo real.

v. Determinar los indicadores del subárea de Triaje para ser mostrados en los reportes propuestos para la gestión de dicha subárea.

vi. Validar y verificar los resultados del sistema. 


\subsection{Justificación y alcance}

Se observa que existe un alto impacto de la tecnología en diversas actividades, es indispensable que el rendimiento de los hospitales y clínicas mejoren con la introducción y asimilación del progreso tecnológico.

Como hospital público, se ha tomado como parte del estudio el área de Emergencia del hospital nacional Cayetano Heredia, institución que proporcionó la información requerida para el proyecto con el que se busca beneficiar a todos los involucrados del proceso de atención de la unidad de Triaje. Por ejemplo, el paciente podrá identificarse únicamente con su número de DNI, recabándose los datos relevantes del paciente para derivarlo a la unidad correspondiente, según su nivel de prioridad, sin tener que volver a proporcionar sus datos. El área de Emergencia mantendrá a las subáreas integradas, al compartirse una base de datos común a la cual accederán todos los involucrados en el proceso de atención, permitiéndoles conocer el estado y realizar un seguimiento del paciente. El proyecto abordará esta problemática interna que se evidencia en la subárea de Triaje a través del tiempo de demora en la atención al paciente; además, podrá consultar el estado del Seguro Integral de Salud (SIS), que es el principal seguro con el que trabajan los hospitales públicos. Para el proyecto se consideró lo siguiente:

- Revisar el enfoque y la definición de la situación actual del hospital nacional Cayetano Heredia en comparación con la situación planteada en la presente propuesta.

- Definir un modelo de negocio que permita el entendimiento de los aspectos más importantes de un hospital público.

- Realizar un análisis preliminar de la factibilidad económica y técnica del proyecto.

- Definir una metodología que abarque las mejores prácticas para el desarrollo del software.

\subsection{Aportes}

Se pone al alcance del Cayetano Heredia, y de los demás hospitales públicos, un sistema web basado en un enfoque de procesos que permita mejorar el nivel de comunicación entre las diferentes subáreas (medicina, ginecología, obstetricia, cirugía, consultorio, caja y admisión) que están relacionadas con la subárea de Triaje. Además, brindar indicadores relevantes y oportunos para la gestión de un hospital público, así como gráficos estadísticos de los indicadores para el personal gerencial y administrativo (patologías más frecuentes, incidentes recurrentes, entre otros). 


\section{Metodología}

\subsection{Modelo de negocio del hospital público}

Con la información obtenida del personal involucrado se desarrolló el siguiente modelo de negocio, denominado modelo Canvas (Osterwalder, Pigneur y Clark. 2010):

\section{Figura 3. Modelo de negocio Canvas para el hospital público}

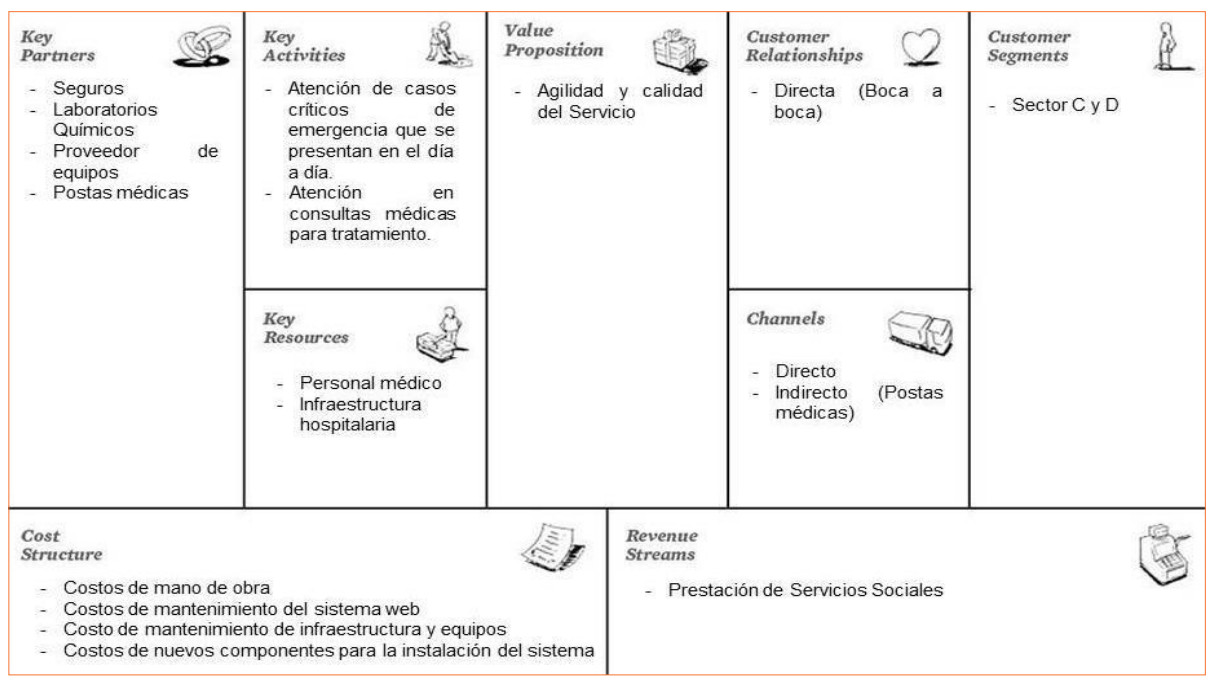

Elaboración propia

En relación a los clientes o usuarios, estos provienen principalmente de los sectores socioeconómicos C y D. El hospital público cuenta con profesionales comprometidos con su trabajo y con la infraestructura hospitalaria debidamente equipada. Si la prestación de servicio es ágil y de calidad, esta sería una propuesta de valor de la institución. La institución principalmente dispone de un canal de distribución directo, donde el paciente solicita el servicio de atención; mientras que el canal indirecto se da a través de las postas médicas que brindan atención a los pacientes de prioridad 3 y 4 . Es justamente con estas atenciones que se tiene ciertos flujos de ingreso, que se dan en el caso de que un paciente no sea asegurado y que necesite de una cirugía o quizás una operación.

Se consideran actividades claves a la atención del paciente en situaciones críticas y a la atención de consultas médicas en las que se brinda un diagnóstico o tratamiento; mientras que los socios claves son las empresas aseguradoras, laboratorios químicos, proveedores de equipos, así como las postas médicas que tienen un convenio con el hospital. Finalmente, para la estructura de costos se toman en cuenta los costos fijos y variables, la infraestructura de TI, el sueldo del personal (que va conectado al servicio que se brinda y el costo en medicinas). 


\subsection{Metodología RUP}

En el desarrollo de la solución se empleó la metodología RUP (Rational Unified Process) que proporciona las mejores prácticas de desarrollo de software y documentación de sistemas orientados a objetos, la cual está compuesta por cuatro fases secuenciales:

- Inicio: Define el alcance del proyecto, presenta una visión general de los requerimientos y la propuesta a realizar.

- Elaboración: Desarrolla los diagramas de caso de uso para definir la arquitectura del sistema a nivel de software, así como el diagrama de red para tener una visión a nivel de hardware de la propuesta de solución.

- Construcción: Explica al detalle la funcionalidad del sistema, dando paso a los casos de uso divididos de acuerdo a las propiedades del mismo.

- Transición: Asegura la disponibilidad del software a través de pruebas y verificación.

Según Torres (2013, p.2), el RUP se basa en "desarrollo iterativo, administración de requerimientos, uso de arquitectura basada en componentes, modelamiento visual, verificación continua de la calidad y administración del cambio".

\subsection{Arquitectura de software y hardware}

La arquitectura de software se refiere a un conjunto de componentes que conforman un sistema, sus interrelaciones y los principios de su diseño. Mowbray y Lilly (1999), citando a Bass, Clements y Kazman, señala que se refiere a las estructuras del sistema conformadas por componentes, sus propiedades visibles y las formas en que estos interactúan y coordinan para alcanzar los objetivos del sistema.

La arquitectura de software presenta componentes lógicos (códigos de programación), mientras que la arquitectura de hardware presenta componentes físicos.

\subsubsection{Model View Controller (MVC)}

De acuerdo a Selfa, Carrillo y Boone (2006), los patrones de arquitectura de software permiten capturar conocimiento de soluciones exitosas en su desarrollo; para el presente proyecto se utilizó el patrón de software Modelo Vista Controlador (Model View Controller, MVC), el cual divide el sistema en tres componentes: el modelo, la vista y el controlador. El uso de MVC permite la reutilización de código, porque la misma información que se requiere para determinado perfil también es requerida por otro usuario, la mayoría de veces. En el presente caso se reutilizaron las vistas, tales como gráficos estadísticos o la opción de listar las atenciones pendientes en 
las diferentes unidades. EI MVC proporciona una mayor claridad del diseño y de las relaciones con los componentes.

\subsubsection{Tecnología ASP.NET}

La tecnología utilizada fue la ASP.NET, framework para aplicaciones web desarrollado por Microsoft, la cual abarca los servicios para crear aplicaciones web escalables y con rendimiento mejorado. ASP.NET incluye: marcos de trabajo (página y controles, servicios Web XML), compilador, infraestructura de seguridad, administración de estado, opciones de configuración, supervisión de estado y características de rendimiento, depuración, entorno de host extensible, administración del ciclo de vida de las aplicaciones y entorno de diseñador extensible (Microsoft, 2007).

\subsubsection{SONA}

La arquitectura de red orientada a servicios (Service-Oriented Network Architecture, SONA), se divide en tres capas principales que se encuentran conectadas entre sí:

- Infraestructura física: Capa que contempla todos los componentes interconectados (servidores, almacenamiento, entre otros).

- Núcleo de servicios comunes: Capa que permite la asignación de los recursos a las aplicaciones y procesos de negocio a través de la infraestructura de red.

- Aplicaciones: Capa de las aplicaciones del negocio y los servicios de colaboración.

\section{Propuesta de solución}

\subsection{Modelo de procesos (AS-IS)}

Se procedió a analizar la situación actual del proceso de atención en el área de Emergencias del hospital, que considera a todas las áreas involucradas. El diagrama del actual proceso de atención se muestra a continuación: 
Figura 4. Modelo de procesos AS-IS

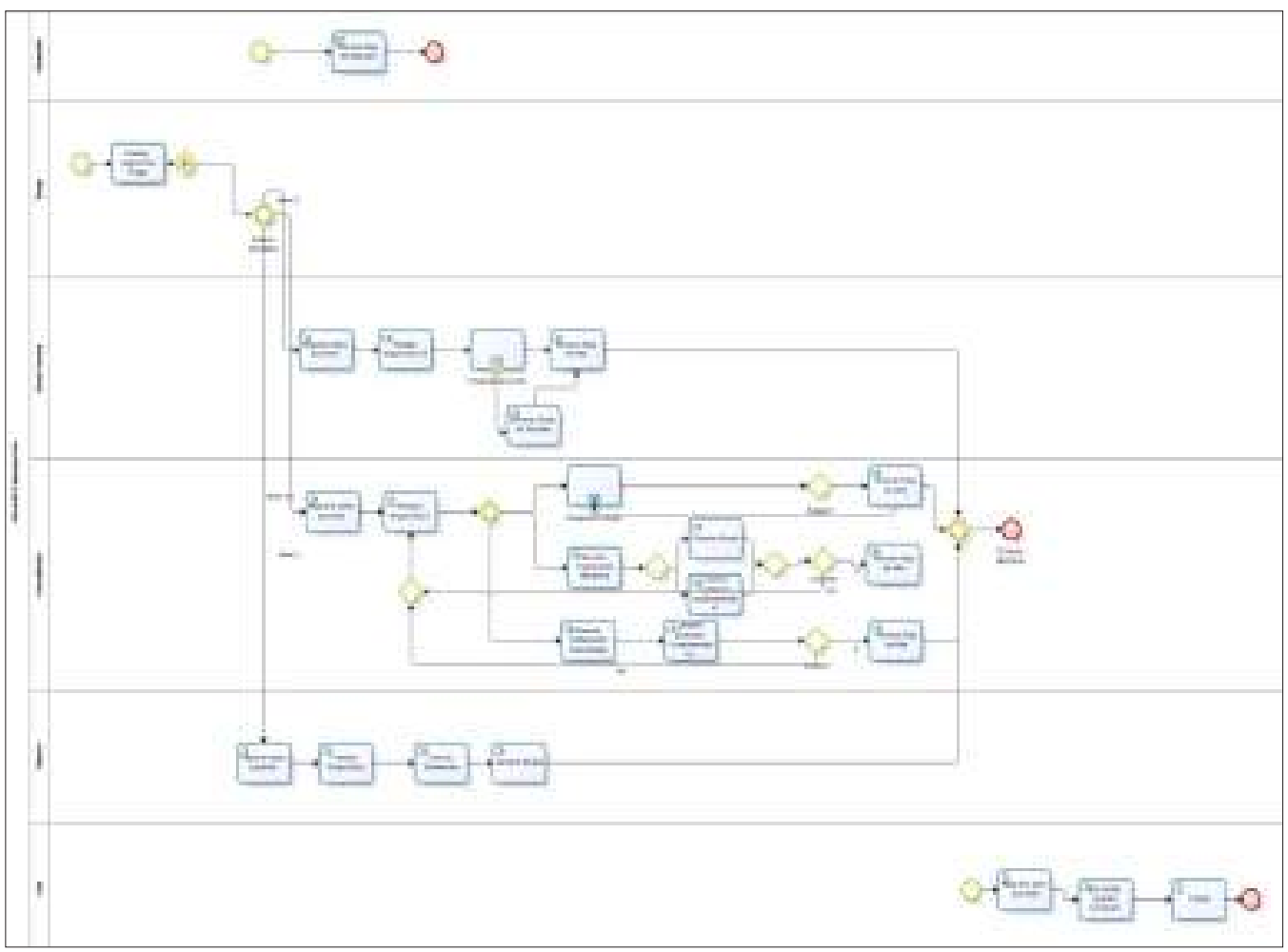

Elaboración propia

El diagrama de procesos AS-IS muestra las dificultades y pasos reiterativos que enfrenta un paciente para ser atendido en el área de Emergencia.

\subsection{Identificación de causas raíz de problema}

La identificación principal del problema, que es la insatisfacción del paciente, se observa en el diagrama de causa-efecto (ver figura 5). 
Figura 5. Diagrama de causa-efecto

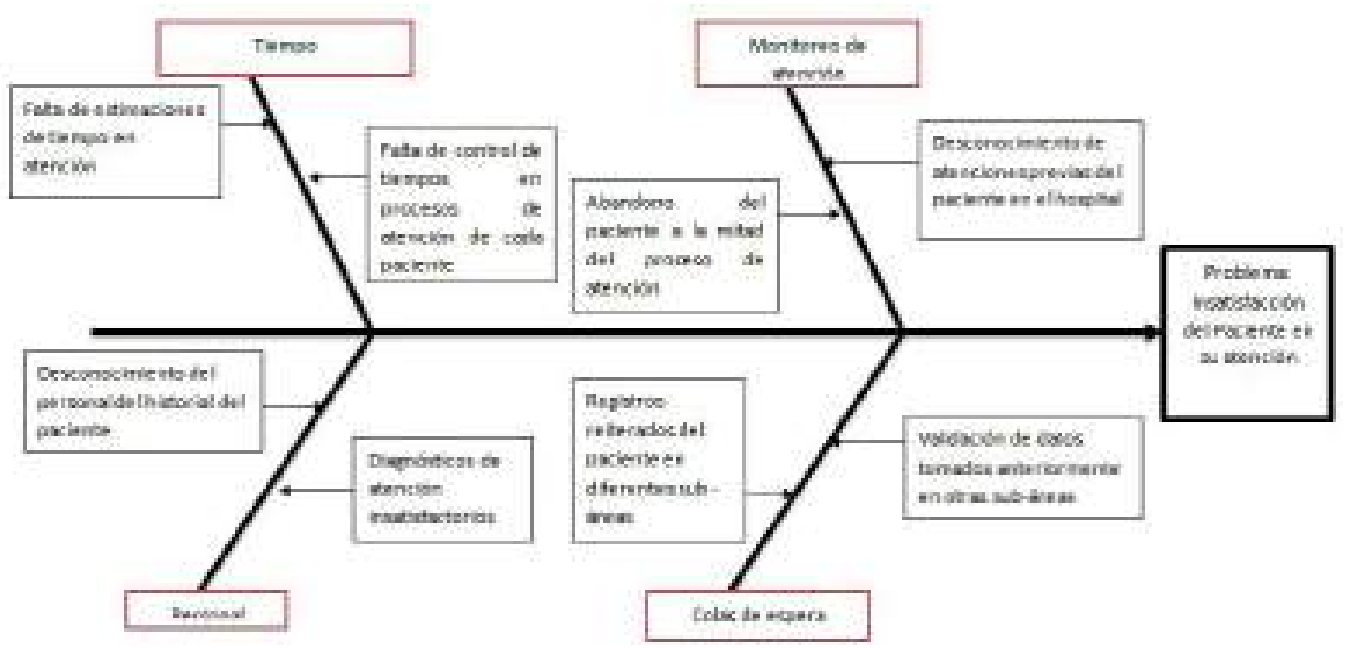

Elaboración propia

La gran mayoría de las causas identificadas se encuentran asociadas al registro manual de los datos de los pacientes, los reiterativos registros, el desconocimiento por parte del personal médico de las enfermedades previas y de las atenciones o recomendaciones que se le brindaron al paciente, entre otros. Por lo tanto, el disponer de un formato consolidado y de alta disponibilidad con la información de los pacientes contribuiría a que ellos estén satisfechos.

\subsection{Propuesta de mejora del proceso de atención de los pacientes}

De acuerdo a la problemática descrita, para el diseño del proceso mejorado se detectaron y eliminaron "cuellos de botella" originados por el registro manual de los datos y por la búsqueda o consolidación de información. A continuación se presenta el diagrama de proceso de atención mejorado que servirá de base para la automatización del mismo en el sistema web: 
Figura 6. Modelo de procesos TO-BE

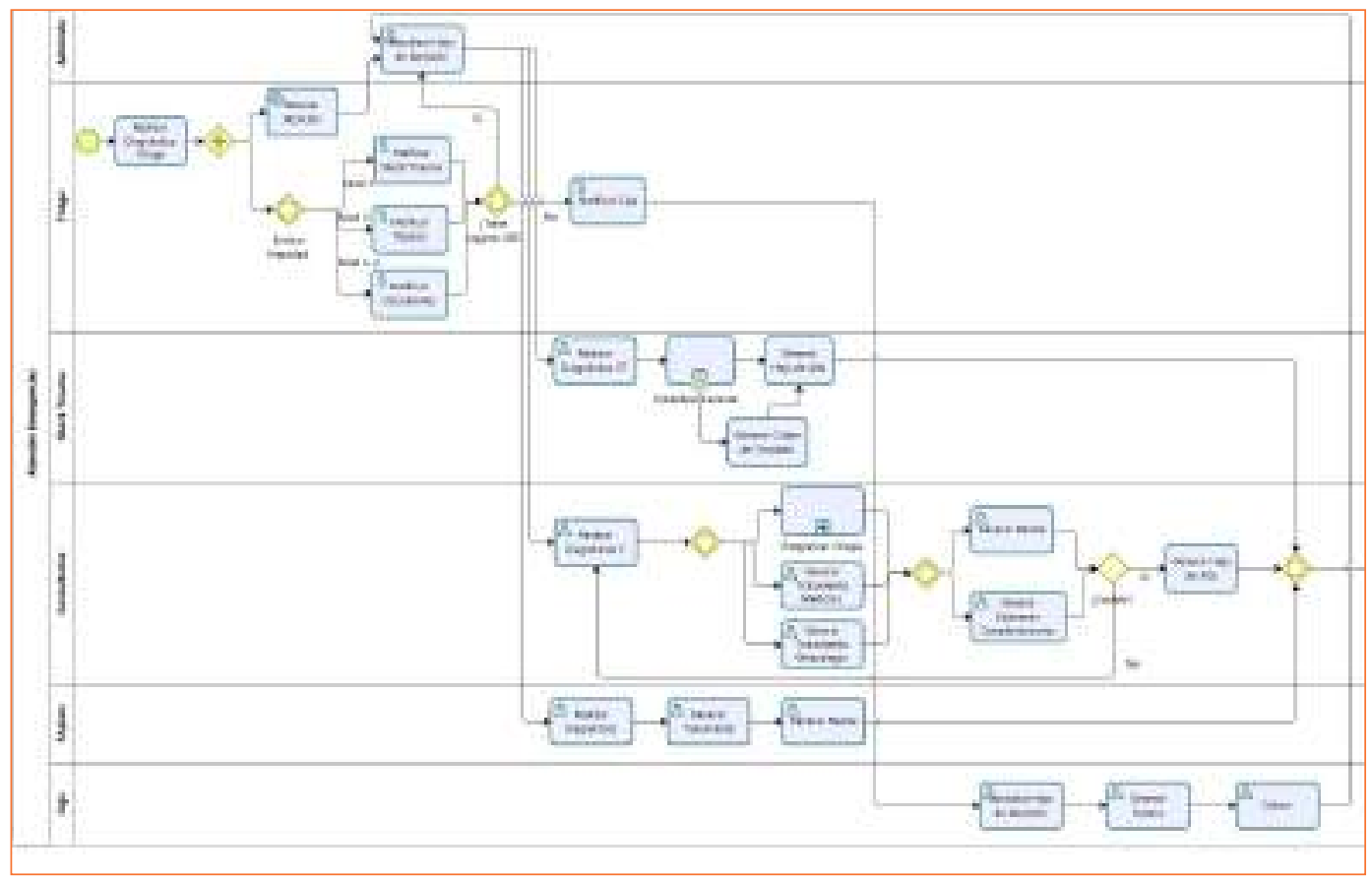

Elaboración propia

La propuesta se sustentará en base a los artefactos requeridos por la metodología RUP, necesarios para llevar a cabo la solución. A continuación se especifican las mejoras del proceso de atención con el sistema web implementado:

- Generación de hojas de atención virtuales: Las atenciones y diagnósticos serán registrados en el sistema web para una posterior consulta.

- Notificación a las subáreas de Emergencia: A través del sistema web se visualizarán los pacientes que sean clasificados por la subárea de Triaje y que, de acuerdo a su patología, serán designados a alguna subárea para su atención.

- Eliminación de duplicidad de actividades: Toda atención se registrará mediante el sistema web y los procesos que se hacían independientemente por cada subárea de Emergencia serán integrados en uno solo, centralizando la información del paciente en un único documento virtual. 


\subsection{Identificación de riesgos asociados al proyecto}

En la siguiente tabla se muestra el análisis de los riesgos a los que puede estar expuesto el sistema web y su nivel de impacto; también se presentan las diversas estrategias que podrían mitigar los riesgos, de tal forma que se reduzca su probabilidad de ocurrencia.

\section{Tabla 2. Riesgos asociados}

\begin{tabular}{|c|c|c|c|c|c|}
\hline$\#$ & Riesgo & $\begin{array}{c}\text { Impacto } \\
(1-10)^{\star}\end{array}$ & $\begin{array}{c}\text { Probabilidad } \\
\text { Ocurrencia }\end{array}$ & $\begin{array}{c}\text { Estrategia } \\
\text { de mitigación de } \\
\text { riesgo }\end{array}$ & Costos \\
\hline 1 & $\begin{array}{l}\text { Equipos de } \\
\text { cómputo que no } \\
\text { estén funcionando } \\
\text { correctamente }\end{array}$ & 4 & $60 \%$ & $\begin{array}{l}\text { Realizar una revisión } \\
\text { quincenal de los } \\
\text { equipos de cómputo } \\
\text { utilizados por el } \\
\text { personal del hospital }\end{array}$ & $\begin{array}{l}\text { Costo por mantenimiento: } \\
\text { S/ } 600 \text { a que incluye: } \\
\text { - Limpieza del sistema } \\
\text { - Mantenimiento del } \\
\text { hardware }\end{array}$ \\
\hline 2 & $\begin{array}{l}\text { Servidor de } \\
\text { aplicaciones } \\
\text { saturado }\end{array}$ & 3 & $20 \%$ & $\begin{array}{l}\text { Revisar conectividad } \\
\text { del servidor } \\
\text { de aplicaciones }\end{array}$ & $\begin{array}{l}\text { Costo por mantenimiento } \\
20 \% \text {; costos de } \\
\text { adquisición del servidor. } \\
\text { Además se sugiere el } \\
\text { soporte del equipo de } \\
\text { sistemas del hospital. }\end{array}$ \\
\hline 3 & $\begin{array}{l}\text { Falla } \\
\text { de conectividad } \\
\text { en la red } \\
\text { del hospital }\end{array}$ & 8 & $10 \%$ & $\begin{array}{l}\text { Contactarse con } \\
\text { un personal de la } \\
\text { operadora para la } \\
\text { solución inmediata } \\
\text { del problema }\end{array}$ & $\begin{array}{l}\text { Sin costo, dado el } \\
\text { contrato entre el hospital } \\
\text { y la operadora. }\end{array}$ \\
\hline 4 & $\begin{array}{l}\text { Pérdida o robo } \\
\text { de información } \\
\text { importante para el } \\
\text { hospital }\end{array}$ & 9 & $15 \%$ & $\begin{array}{l}\text { Tener un respaldo de } \\
\text { la información en un } \\
\text { registro histórico que } \\
\text { se puede realizar de } \\
\text { manera mensual }\end{array}$ & $\begin{array}{l}\text { Costo por back-up a } \\
\text { la base de datos del } \\
\text { sistema: S/ } 100^{\star} . \\
\text { Se sugiere el apoyo del } \\
\text { equipo de sistemas del } \\
\text { hospital. }\end{array}$ \\
\hline 5 & Fallo de UPS & 8 & $10 \%$ & $\begin{array}{l}\text { Mantenimiento } \\
\text { preventivo }\end{array}$ & $\begin{array}{l}\text { Se sugiere el apoyo del } \\
\text { equipo de sistemas del } \\
\text { hospital. }\end{array}$ \\
\hline 6 & $\begin{array}{l}\text { Pérdida en la } \\
\text { conexión de la } \\
\text { base de datos } \\
\text { con el sistema } \\
\text { web }\end{array}$ & 9 & $40 \%$ & $\begin{array}{l}\text { Realizar consultas } \\
\text { de prueba antes del } \\
\text { funcionamiento de } \\
\text { la base }\end{array}$ & $\begin{array}{l}\text { Se sugiere el apoyo del } \\
\text { equipo de sistemas del } \\
\text { hospital. }\end{array}$ \\
\hline
\end{tabular}

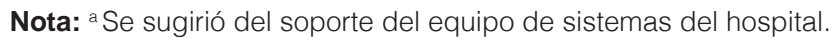
Elaboración propia

Para la mitigación de los riesgos señalados se requiere del soporte del área de sistemas del hospital. 


\subsection{Metodología de desarrollo}

Para desarrollar la solución se utilizó la metodología de RUP (Rational Unified Process). A continuación se detalla el desarrollo en las diferentes fases de la misma.

\subsubsection{Fase de inicio}

En la propuesta de mejora TO-BE, figura 6, sobre la cual se basó el sistema web, se eliminaron las actividades reiterativas en el proceso de atención y se sustituyeron parte de las actividades manuales por procedimientos automáticos. En esta fase se determinaron los requerimientos funcionales y no funcionales (ver tablas 3 y 4).

\section{Tabla 3. Requerimientos funcionales}

\begin{tabular}{|c|c|}
\hline Código & Descripción \\
\hline RF01 & $\begin{array}{l}\text { Buscar paciente: Realizar la búsqueda del paciente mediante DNI, a través del sistema } \\
\text { web. }\end{array}$ \\
\hline RFO2 & $\begin{array}{l}\text { Buscar atenciones asignadas: Al iniciarse una atención, las subáreas involucradas tendrán } \\
\text { la capacidad de poder buscar los pacientes asignados. }\end{array}$ \\
\hline RFO3 & $\begin{array}{l}\text { Generar gráficos: Generación de reportes que se visualizarán con gráficos de diferente } \\
\text { estilo. }\end{array}$ \\
\hline RFO4 & $\begin{array}{l}\text { Buscar historial: El médico podrá visualizar el historial de atenciones que el paciente ha } \\
\text { recibido anteriormente en el hospital. }\end{array}$ \\
\hline RFO5 & Ingreso de sistema web: Inclusión de un usuario y contraseña para el ingreso al sistema. \\
\hline RFO6 & $\begin{array}{l}\text { Buscar atenciones pendientes: Se contará con una lista de atenciones pendientes para } \\
\text { cada una de las subáreas. }\end{array}$ \\
\hline RFO7 & $\begin{array}{l}\text { Seleccionar pacientes: El sistema web permitirá la selección de los pacientes que } \\
\text { deben ser atendidos en caso de que otros pacientes hayan desistido de continuar con } \\
\text { el proceso de atención. }\end{array}$ \\
\hline RF08 & $\begin{array}{l}\text { Registrar atención y diagnóstico: Registro de la atención del paciente y del tratamiento } \\
\text { realizado o el medicamento proporcionado. }\end{array}$ \\
\hline RFO9 & $\begin{array}{l}\text { Atender solicitud: Un paciente que ha pasado por la subárea de Caja y que ha sido } \\
\text { seleccionado, es cambiado de estado para que se registre su atención. }\end{array}$ \\
\hline RF10 & $\begin{array}{l}\text { Registrar recibo: Usado generalmente para el subárea de Caja cuando un paciente ha } \\
\text { realizado el pago de alguna atención o medicamento que haya comprado dentro del } \\
\text { hospital. }\end{array}$ \\
\hline RF11 & Asignar ficha: Ficha que es proporcionada por una de las subáreas de Emergencia. \\
\hline RF12 & Registrar estado: Cambios de estado del paciente por atención realizada en las subáreas. \\
\hline
\end{tabular}




\section{Tabla 4. Requerimientos no funcionales}

\begin{tabular}{|c|c|c|}
\hline Tipo de requisito & Código & Descripción \\
\hline $\begin{array}{l}\text { Restricciones } \\
\text { del diseño }\end{array}$ & RNF-001 & $\begin{array}{l}\text { La aplicación se desarrollará en el lenguaje de programación C\# con } \\
\text { la tecnología de Framework } 3.5 \text { de ASP.NET Web Application }\end{array}$ \\
\hline \multirow{2}{*}{$\begin{array}{l}\text { Interfaces } \\
\text { de usuario }\end{array}$} & RNF-002 & $\begin{array}{l}\text { El diseño de la interfaz se desarrollará utilizando el lenguaje HTML y } \\
\text { estilo en cascada (cascading style sheets, CSS). }\end{array}$ \\
\hline & RNF-003 & La resolución mínima será de 1024×768 píxeles. \\
\hline Seguridad & RNF-004 & $\begin{array}{l}\text { A cada médico o asistente se le asignará un usuario y una clave, de } \\
\text { acuerdo a un perfil determinado. }\end{array}$ \\
\hline \multirow{2}{*}{$\begin{array}{l}\text { Requisitos } \\
\text { del sistema }\end{array}$} & RNF-005 & $\begin{array}{l}\text { El sistema no deberá generar conflicto con las aplicaciones existentes } \\
\text { en la empresa. }\end{array}$ \\
\hline & RNF-006 & $\begin{array}{l}\text { Procesador DualCore o superior, } 2 \text { GB de RAM y disco duro de } 250 \\
\text { GB. }\end{array}$ \\
\hline \multirow{2}{*}{$\begin{array}{l}\text { Requisitos } \\
\text { de desempeño }\end{array}$} & RNF-007 & $\begin{array}{l}\text { Ingreso simultáneo de seis usuarios como mínimo, que son el total de } \\
\text { usuarios que ingresan al crear un proceso de atención. }\end{array}$ \\
\hline & RNF-008 & $\begin{array}{l}\text { El tiempo de respuesta del sistema para operaciones de registro, } \\
\text { búsqueda y visualización de reportes debe ser como máximo de } 10 \\
\text { segundos, con un límite de seis usuarios concurrentes en el mismo. }\end{array}$ \\
\hline Usabilidad & RNF-009 & $\begin{array}{l}\text { Facilidad de uso en cada interface. El sistema remarcará errores de } \\
\text { ingreso de datos y mensajes de ayuda. Además tendrá un buscador } \\
\text { de patologías con propiedad de autocompletado. }\end{array}$ \\
\hline \multirow{3}{*}{ Eficiencia } & RNF-010 & $\begin{array}{l}\text { Se almacenará los códigos estándares utilizados para identificar las } \\
\text { patologías según la CIE (Clasificación Internacional de Enfermedades). }\end{array}$ \\
\hline & RNF-011 & $\begin{array}{l}\text { Se almacenará los datos del paciente que por primera vez llega a una } \\
\text { atención. }\end{array}$ \\
\hline & RNF-012 & $\begin{array}{l}\text { Se asignará automáticamente la prioridad y las unidades administrati- } \\
\text { vas donde deberá dirigirse el paciente. }\end{array}$ \\
\hline \multirow{2}{*}{ Disponibilidad } & RNF-013 & $\begin{array}{l}\text { El sistema contará con un nivel de disponibilidad de alto rango, con } \\
\text { un porcentaje estimado de } 99,70 \% \text {, de tal forma que el sistema no } \\
\text { sobrepasará las } 26 \text { horas al año de indisponibilidad. }\end{array}$ \\
\hline & RNF-014 & $\begin{array}{l}\text { Se tendrá como respaldo a un servidor, con la finalidad de mantener } \\
\text { el sistema web funcionando con el mínimo de fallas. }\end{array}$ \\
\hline
\end{tabular}


Además, se elaboró el diagrama de casos de uso donde se visualizan los módulos del sistema web y las interrelaciones con los sistemas a los que brindará soporte:

\section{Figura 7. Diagrama de caso de uso}

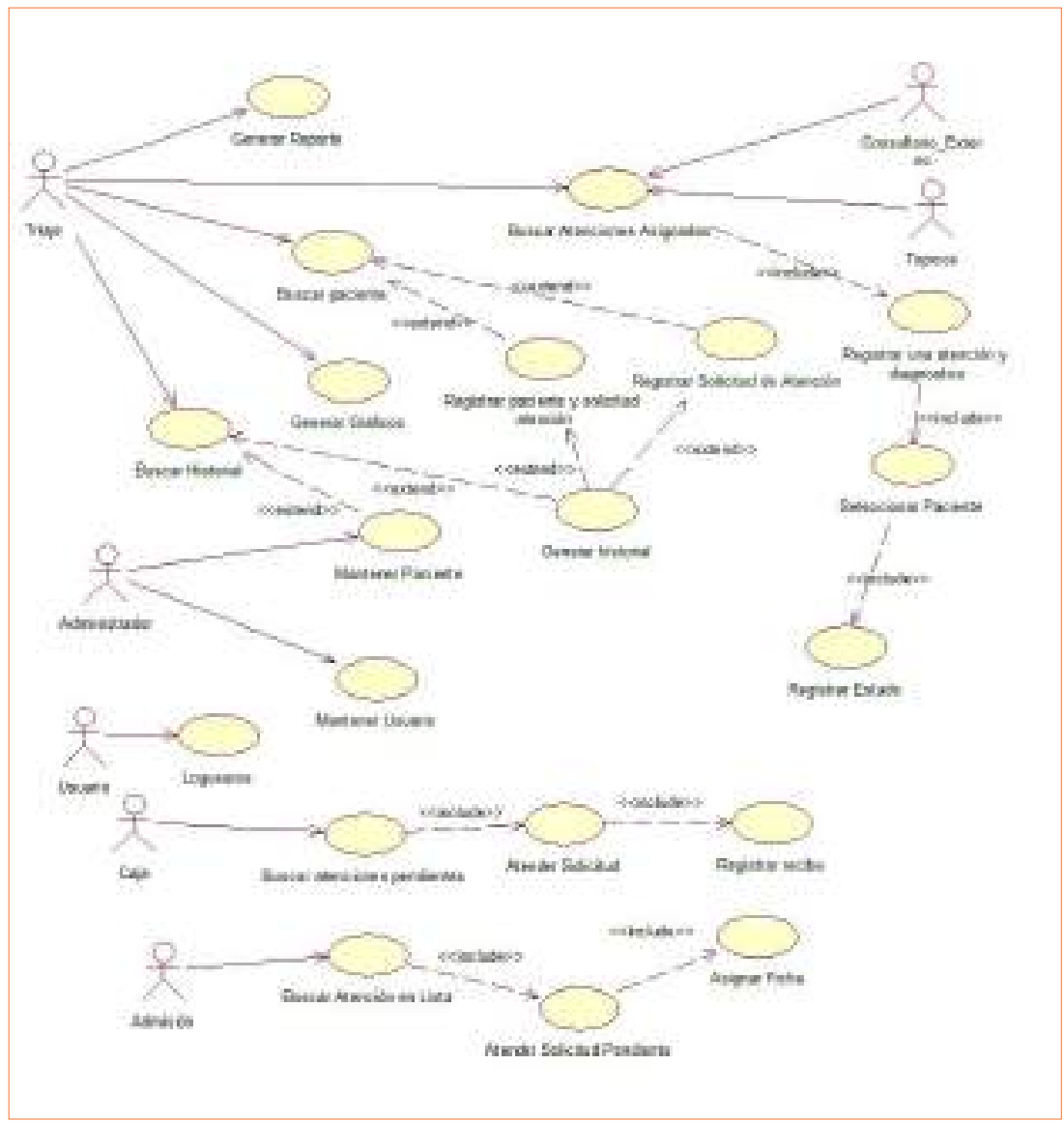

Elaboración propia

\subsubsection{Descripción de principales casos de uso del sistema}

CUS 01: Registro de solicitud de atención del paciente

i. Actor: Médico de Triaje.

ii. Propósito: Registrar la solicitud del paciente que ha sido visto en la unidad de Triaje para que continúe con el proceso de atención.

iii. Breve descripción: Este formulario le permitirá al médico registrar diversos factores que ayuden a mejorar el proceso de atención del paciente. 
iv. Flujo básico de eventos:

1. El médico ingresa el DNI o se identifica al paciente como NN.

2. El sistema web mostrará la interfaz de registro de solicitud del paciente:

- $\quad$ DNI, nombre del paciente, apellido paterno, apellido materno

- Edad

- Distrito

- Sexo

- Tipo de seguro

- Datos de la última atención: estado, descripción del ClE, prioridad, tópico derivado, acompañante, fecha de visita, resumen de la visita

- Ingreso de datos para solicitar una nueva atención: descripción de CIE, doctor asignado, acompañante, observación, destino.

3. Se tiene la opción "ver historial" por la cual el médico podrá visualizar las atenciones anteriores realizadas.

4. Se tiene la opción "crear" para finalizar con la solicitud de atención del paciente y "regresar" para retornar al ingreso del DNI.

5. El médico selecciona la opción "crear".

6. Se muestra un mensaje "Se creó la atención con éxito" y finaliza el caso.

v. Subflujos

1. DNI incorrecto: En el punto 1 del flujo básico si el médico ingresa un DNI menor a ocho dígitos, el sistema web mostrará un mensaje indicándole que el DNI ingresado no es correcto.

2. Paciente NN: En el punto 1, si el médico selecciona la opción "paciente no identificado", en el sistema no se visualizará el ingreso del DNI y se generará un código aleatorio. 
3. Paciente nuevo: En el punto 2, si es la primera vez que el paciente se atiende en el hospital, los datos deberán ser llenados por el médico para que se realice un registro primario de la atención.

4. Destino fuera de emergencia: En el punto 2, cuando se selecciona el lugar de destino donde será derivado el paciente, y no es dentro de Emergencia, el dato no se mostrará en el sistema.

\section{CUS 02: Listar y realizar atenciones pendientes}

- Actor: Médico de Triaje

- Propósito: Listar las atenciones que se tiene pendientes, cuando se realice la atención se deberá especificar el diagnostico final del paciente.

- Breve descripción: Permitirá al médico visualizar las atenciones que le han sido asignadas y tener conocimiento de los datos del paciente, así como otorgarle un estado, el cual se registrara para su uso en próximas atenciones.

- Flujo básico de eventos:

1. El medico visualizará la lista de atenciones seleccionando la opción "atenciones por realizar". El sistema mostrará una tabla donde se visualizan la lista de pacientes por atender, con los siguientes datos:

- $\quad$ Orden, DNI y nombre completo del paciente

- Fecha y hora de la solicitud

- Edad

- Estado

2. El médico selecciona la opción "búsqueda", simbolizada con una lupa, y se muestra una ventana con el detalle del paciente y tres opciones: "atendido", "abandonado" y "rechazado".

3. El médico ingresa el diagnóstico final.

4. El médico selecciona la opción "atendido".

5. Se muestra la confirmación de la acción y las opciones "si" y "no". Si el médico selecciona "sí", el caso de uso finaliza. 
v. Subflujos

1. Abandonado: En el punto 4 del flujo básico, si el médico selecciona "abandonado" se le asigna dicha atención y finaliza el caso de uso.

2. Rechazado: En el punto 4, si el médico selecciona rechazado, se le asigna dicha atención y finaliza el caso de uso.

3. No: En el punto 4, si el médico selecciona esta opción retornará a la ventana de detalle del paciente.

De igual forma se especificaron los siguientes casos de uso: CUS-03 Atender paciente, CUS-04 Atención de solicitud en Caja, CUS-05 Generar reporte.

\subsubsection{Fase de elaboración}

Diagrama de entidad-relación

Para representar las entidades en las cuales se soportará el sistema web, se elaboró el diagrama Entidad-Relación (figura 8), en el que se visualizan las tablas, las relaciones y los atributos del proceso de atención. 
Figura 8. Diagrama de entidad-relación

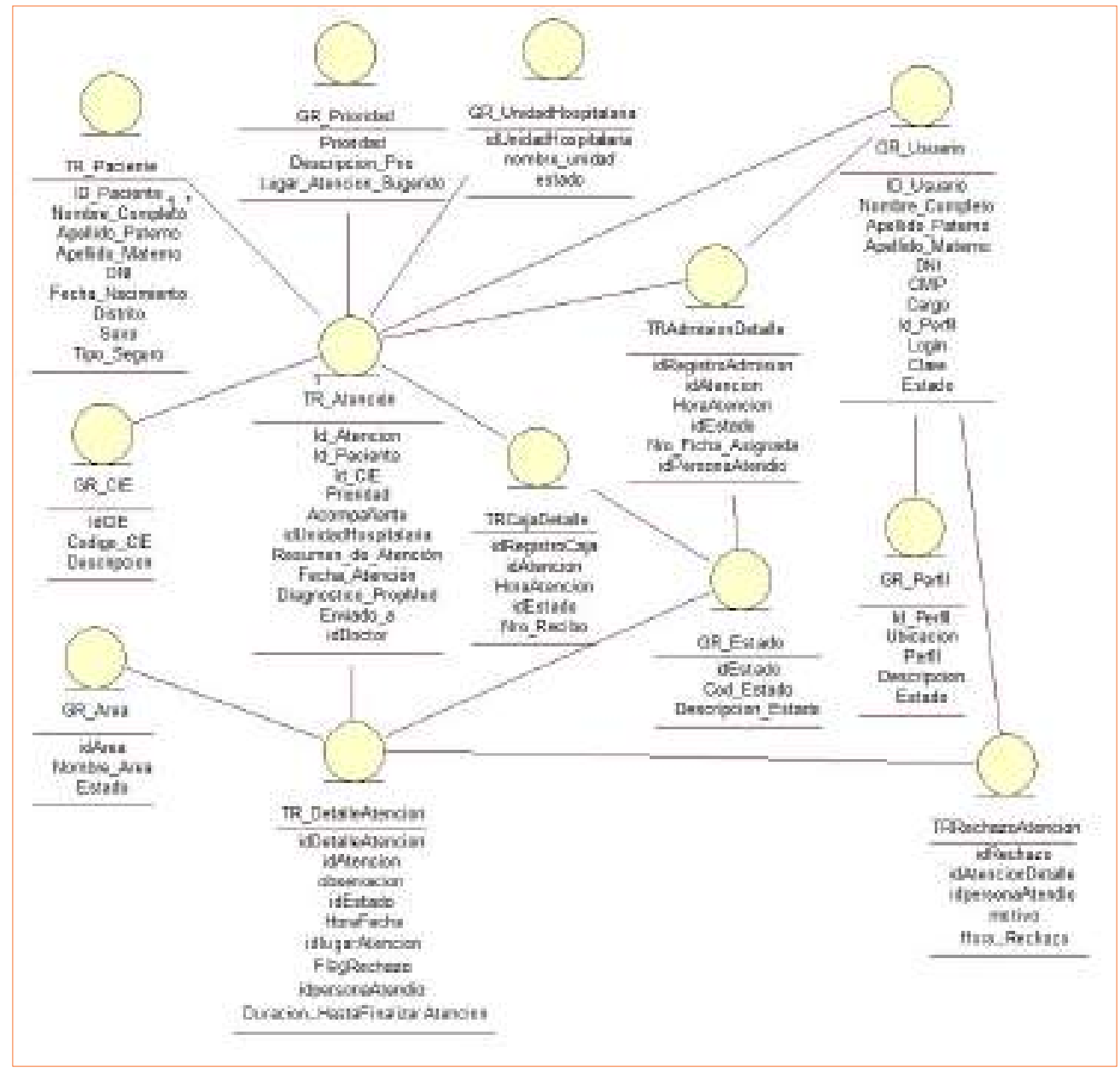

Elaboración propia

General Relationship (GR) hace referencia a tablas maestras en las cuales se guardan datos de negocio, tales como el área de Emergencia, y las unidades hospitalarias, además de la clasificación internacional de enfermedades (CIE). También se aprecian las Transactional Relationship (TR), que son las tablas transaccionales donde se guarda la información que se recopila a diario. En la tabla principal TR_Atencion se centrará el registro de las atenciones realizadas a diario en la unidad de Triaje y será el nexo con las demás tablas. 
Diagrama de secuencia

Para mostrar la interacción de los componentes, se muestran los diagramas de secuencia relacionados al registro de atención y para buscar atenciones pendientes.

Figura 9. Diagrama de secuencia. Registrar una atención.

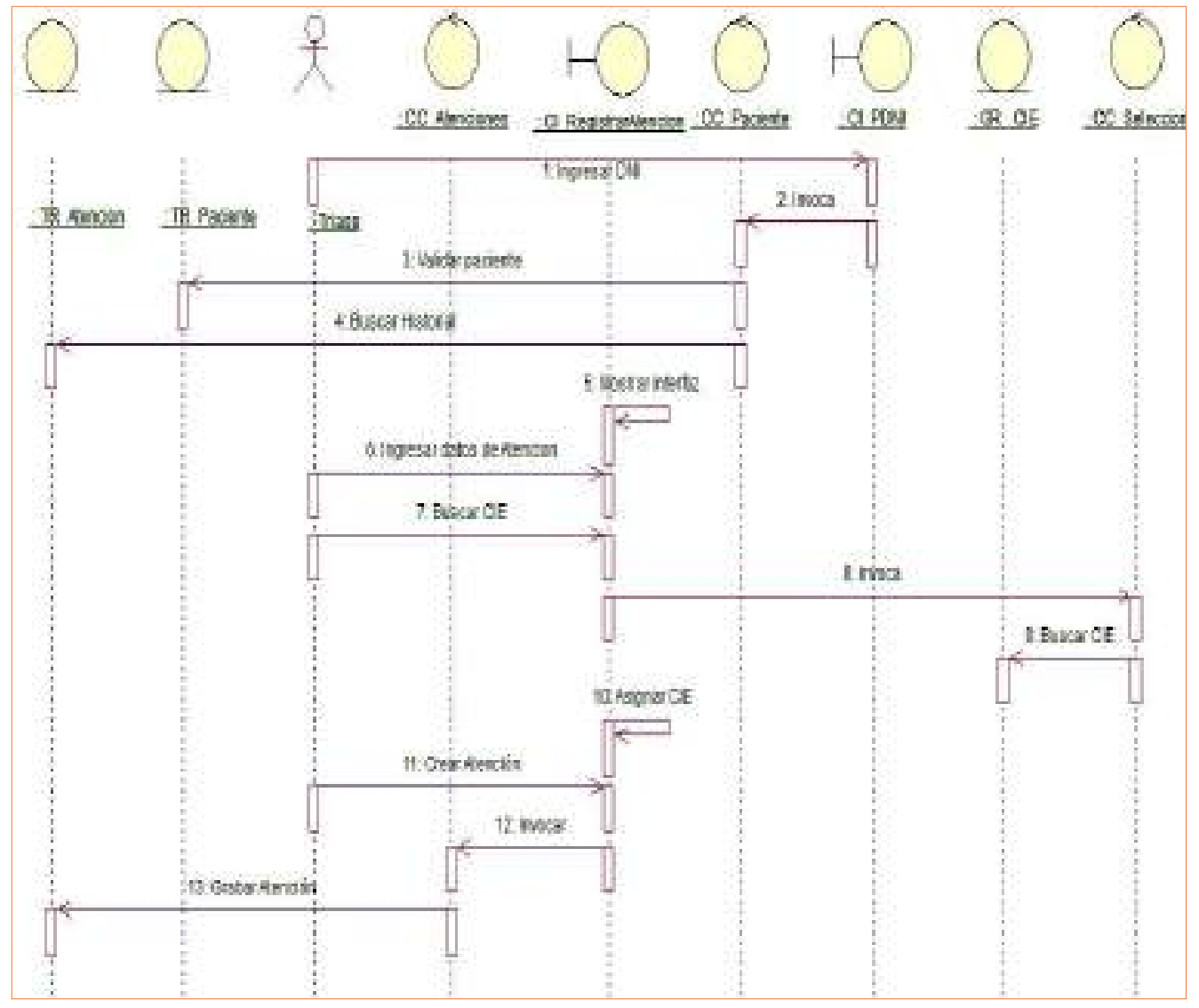

Elaboración propia 
Figura 10. Diagrama de secuencia. Buscar atenciones pendientes

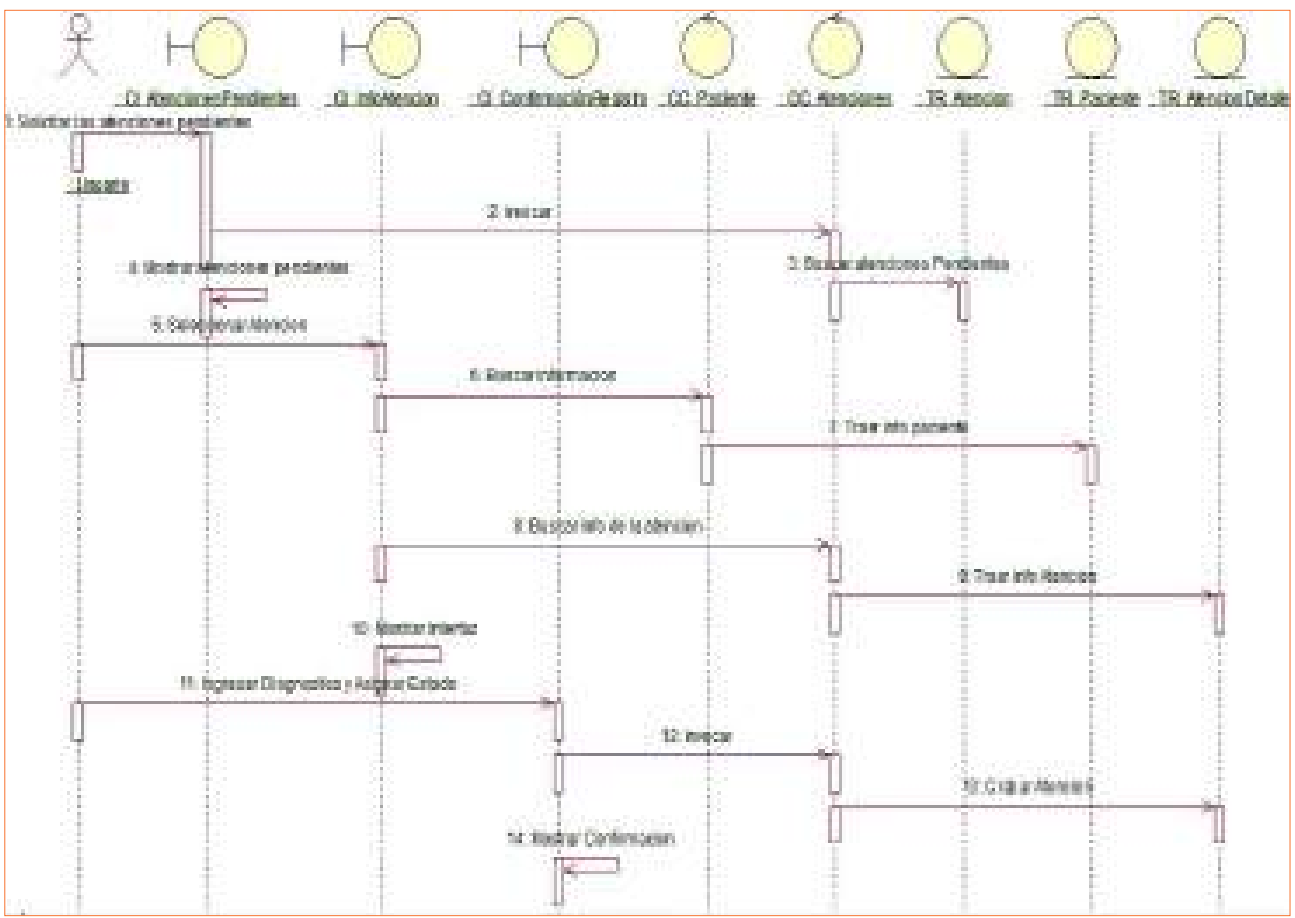

Elaboración propia

Diagrama de paquetes

El diagrama visualiza las relaciones que existen entre los casos de uso, además de permitir su agrupación de acuerdo a su funcionalidad y el proceso que soporta. 
Figura 11. Diagrama de paquetes

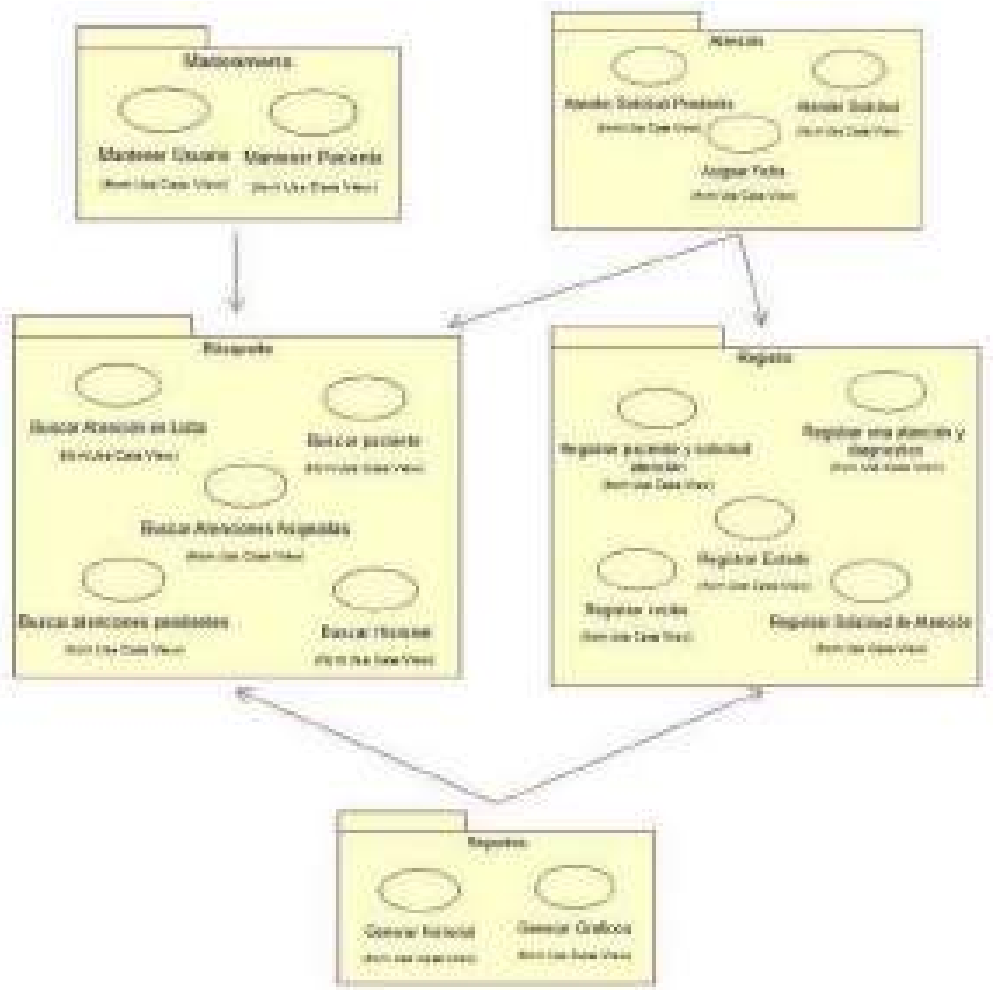

Elaboración propia

Diagrama de red

Debido a que la red actual no proporciona el respaldo adecuado a nivel de switches y servidores que aseguren la disponibilidad del sistema, se propuso un diagrama de red que cumple con los siguientes lineamientos de arquitectura de red orientada a servicios (SONA):

- Tolerancia a fallos: Se cuenta con redundancia en cada componente, de tal manera que, en caso de falla, la disponibilidad del sistema no se verá afectada.

- Seguridad: Acceso a los dispositivos de red mediante validación del usuario y clave del active directory. Adicionalmente, contará con seguridad de firewall.

- Calidad de servicio: Al ser un sistema de información, no se requerirá de transmisiones ininterrumpidas de voz y video.

- Escalabilidad: Se maneja una cantidad limitada de usuarios, por lo que no requeriría que la red sea escalable. 
Figura 12. Diagrama de red. SONA

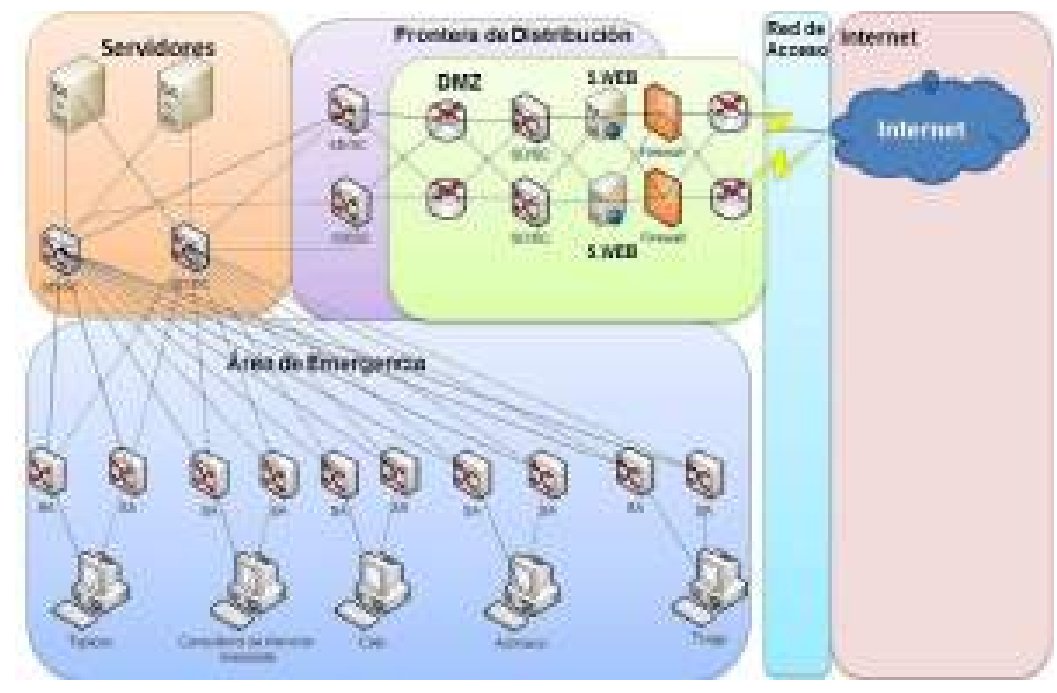

Elaboración propia

Sin embargo, el diagrama mostrado requiere de componentes con costos de hardware elevados; por ello, se elaboró una propuesta acorde al presupuesto de un hospital público, con las mejores prácticas de infraestructura y con alta disponibilidad.

Figura 13. Diagrama de red

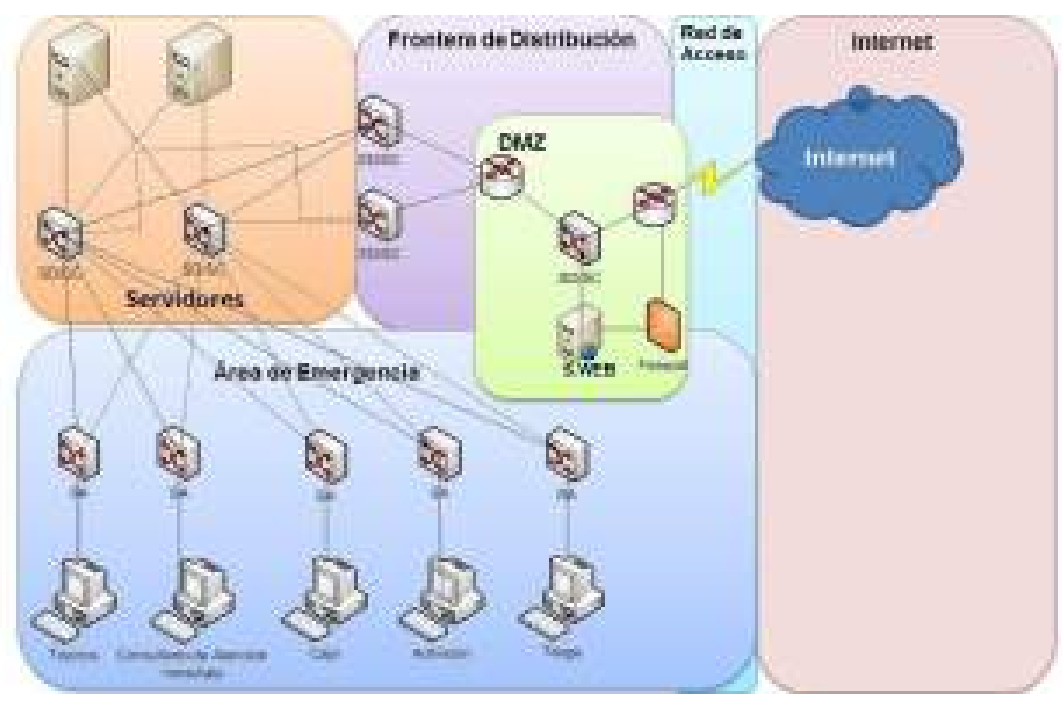

Elaboración propia 
Cada computadora cuenta con un switch de acceso (SA), sin redundancia; sin embargo, se contarán con switches de backup en almacén para asegurar la disponibilidad del sistema. Estos switches estarán enlazados a los switches de distribución (SD), que también cumplen la función de switch de la capa de núcleo, denominado switch core (SC), en el bloque de servidores donde se necesitará al menos dos servidores (principal y de respaldo). Estos switchs de distribución/capa de núcleo se encuentran representados como SD/SC en dicha figura.

El sistema no requiere de conexión a Internet, ya que no se realizarán consultas externas ni será accedido fuera de los límites del hospital; no obstante, sí se requerirá para las actualizaciones de los componentes de software, con lo cual se adiciona al diagrama la frontera de distribución, que enlazará al bloque de servidores con la zona desmilitarizada (DMZ) a través de un switch de distribución/capa de núcleo (SD/SC), permitiendo que el DMZ se enlace a Internet mediante la red de acceso. Los enlaces con los que cuenta el hospital son enlaces de cobre de tipo "par trenzado no blindado" (Unshielded Twisted Pair, UTP), de categoría 5e, que otorgan una capacidad de transmisión de hasta 1 GB por segundo. Dado que no existen mediciones previas del tráfico de datos en el hospital, se realizaron mediciones cuando el sistema web fue instalado y consolidado.

\section{Estudio de factibilidad}

\subsection{Factibilidad técnica o tecnológica}

Se realizó un levantamiento de información sobre los equipos de cómputo con prestaciones aceptables para la implementación del sistema web propuesto; en la mayoría de hospitales se cuenta con computadoras que son usadas para el manejo de paquetes ofimáticos y que por sus características pueden ser utilizadas para el sistema web propuesto.

Las características de las computadoras disponibles fueron las siguientes: memoria RAM-de 2 GB, disco duro de 250 GB, teclado y mouse operativos, sistema operativo Windows 7 de 64 bits, y browser Internet Explorer 8; con lo que cumplen con los requerimientos mínimos especificados en el RNF-006. Además se efectuó un análisis de la infraestructura disponible en comparación con lo mínimo requerido, según se detalla en la arquitectura de red (ver figura 13). A partir de los análisis realizados se elaboró la siguiente tabla con los recursos requeridos: 
Tabla 5. Recursos requeridos en la arquitectura de red

\begin{tabular}{|c|c|c|c|c|}
\hline Tipo & $\begin{array}{l}\text { Cantidad } \\
\text { requerida }\end{array}$ & Ítem & Disponibilidad & Por adquirir \\
\hline \multirow{7}{*}{ Hardware } & 10 & $\mathrm{PC}^{1}$ & 10 & 0 \\
\hline & 11 & Switch & 8 & 3 \\
\hline & 2 & Router & 2 & 0 \\
\hline & 2 & Servidor de aplicaciones & 1 & 1 \\
\hline & 1 & Servidor web & 1 & 0 \\
\hline & 2 & Servidor de base de datos & 1 & 1 \\
\hline & 2 & $\begin{array}{l}\text { Unidad de respaldo de energía } \\
\text { (UPS) }\end{array}$ & 1 & 1 \\
\hline \multirow{3}{*}{ Software } & 2 & Licencia Windows Server 2008 & 1 & 1 \\
\hline & 2 & $\begin{array}{l}\text { Licencia motor de base de datos } \\
\text { SQL Server } 2008\end{array}$ & 2 & 0 \\
\hline & 10 & Antivirus & 10 & 0 \\
\hline
\end{tabular}

Elaboración propia

Como se demuestra, el hospital dispone parcialmente de los recursos y necesita adquirir otros. En algunos casos se están obteniendo recursos como contingencia ante posible falla de los equipos; por ejemplo, adquirirá un switch adicional.

\subsection{Factibilidad económica}

\subsubsection{Costo de hardware y software}

De acuerdo con la arquitectura de software y redes se determinaron los costos asociados a los recursos faltantes, como se expone a continuación:

1 Compuesto por monitor, CPU, mouse y teclado. 
Tabla 6. Costos de hardware y software

\begin{tabular}{|c|c|c|c|c|}
\hline Ítem & Sizing & $\begin{array}{l}\text { Precio } \\
\text { unitario } \\
\text { (S/) }\end{array}$ & Cantidad & $\begin{array}{l}\text { Precio total } \\
\text { (S/) }\end{array}$ \\
\hline Switch & $\begin{array}{l}\text { Cisco SG300-10 10-port Gigabit } \\
\text { Managed Switch }\end{array}$ & 533,6 & 3 & 1600,8 \\
\hline $\begin{array}{l}\text { Servidor } \\
\text { de aplicaciones }\end{array}$ & \multirow{2}{*}{$\begin{array}{l}\text { Servidor HP Proliant ML } 110 \text { g7-Xe- } \\
\text { on E3 } 1220 \text { Quad core } 3.1 \text { GHz- } 8 \\
\text { GB RAM }\end{array}$} & 2279,0 & 1 & 2279,0 \\
\hline $\begin{array}{l}\text { Servidor de base } \\
\text { de datos }\end{array}$ & & 2279,0 & 1 & 2279,0 \\
\hline UPS & $\begin{array}{l}\text { Emerson GXT2-2000RT120 Liebert } \\
2000 \text { VA - 120V }\end{array}$ & 580,0 & 1 & 580,0 \\
\hline \multirow[t]{2}{*}{$\begin{array}{l}\text { Windows Server } \\
2008\end{array}$} & Licencia para 10 clientes & 442,0 & 1 & 442,0 \\
\hline & & & Costo total & $\mathrm{S} / 7180,8$ \\
\hline
\end{tabular}

Elaboración propia

\subsubsection{Costo de mantenimiento}

Para el mantenimiento y modificación del sistema web, se tuvo en cuenta el apoyo de los creadores e impulsadores del proyecto por un plazo no mayor a un año, quienes se encargan de verificar los inconvenientes del sistema; posteriormente se capacitará al personal del área de Informática del hospital para que lleven a cabo las modificaciones que se requieran. Se han considerado los siguientes costos, conforme al nivel de complejidad de la modificación o inclusión requeridas:

\section{Tabla 7. Costos de mantenimiento}

\begin{tabular}{|l|l|r|}
\hline Nivel & \multicolumn{1}{|c|}{ Descripción } & Costo aproximado (S/) \\
\hline Nivel 1 & Actualizaciones de código & 4520 \\
\hline Nivel 2 & Modificación o agregación de características al módulo & 9040 \\
\hline Nivel 3 & Adición de nuevos módulos en el sistema web & 15066 \\
\hline & Costo total & S/ 28626 \\
\hline
\end{tabular}

Elaboración propia 


\section{Verificación y validación}

\subsection{Pruebas unitarias de verificación}

Se hicieron las siguientes pruebas unitarias de software con el fin de verificar si se obtuvieron los resultados esperados en los principales métodos del flujo de atención en Triaje:

a) Prueba unitaria: Buscar paciente.

Identificador: SIGEST-TEST-0001

Caso de uso: Buscar paciente.

Objetivo: Verificar que el sistema web retorne los datos del paciente.

Método: Datos paciente Test

Descripción: Se selecciona el paciente y se oprime el botón editar.

Resultado esperado: Retorna los datos del paciente (nombre completo, sexo, edad, distrito, tipo de seguro).

b) Prueba unitaria: Registrar atención.

Identificador: SIGEST-TEST-0002

Caso de uso: Registrar atención y diagnóstico.

Objetivo: Verificar que el sistema web retorne un resultado exitoso tras la inserción de atención.

Método: Historial Controller Test, crear Atención Test.

Descripción: Ingresar los datos obligatorios y luego oprimir el botón "Registrar".

Resultado esperado: Retorne el valor 1, el cual indica que se grabó correctamente el registro de atención.

c) Prueba Unitaria: Buscar atenciones pendientes.

Identificador: SIGEST-TEST-0003

Caso de uso: Buscar atenciones pendientes.

Objetivo: Verificar que el sistema web retorne la lista de pacientes con pendiente de atención.

Método: Atenciones Controller Test, Listar atenciones por realizar Test.

Descripción: Se deja el campo DNI y se oprime el botón buscar. 
Resultado esperado: Lista y detalle de pacientes pendientes de atención: (nombre, DNI, fecha y hora de la solicitud, edad y estado).

d) Prueba Unitaria: Generar gráficos.

Identificador: SIGEST-TEST-0004

Caso de uso: Generar gráficos.

Objetivo: Verificar que el sistema web retorne la data a mostrar en los gráficos estadísticos.

Método: Gráfico Controller test, Traer Info Gráfico01 Test.

Descripción: Se selecciona el gráfico top 10 de patologías más frecuentes.

Resultado esperado: Gráfico con las 10 patologías frecuentes.

e) Prueba Unitaria: Ingreso de sistema.

Identificador SIGEST-TEST-0005

Caso de Uso Ingreso de sistema.

Objetivo: Verificar que el sistema web valide si el usuario y contraseña.

Método Usuario Controller Test, Validar Usuario Test.

Descripción: Introducir el usuario y contraseña correcta.

Resultado esperado: Obtener el perfil, nombre e ID del usuario de la sesión.

Para todas las pruebas realizadas se obtuvieron resultados satisfactorios.

\subsection{Validación}

Para la validación del sistema web se consideró como escenario un flujo normal de atención en el área de Emergencia del Cayetano Heredia para un paciente sin seguro SIS, con dolor abdominal y atendido el sábado durante la mañana. Luego de realizada la atención se simuló las mismas condiciones a través del sistema y se efectuaron las siguientes tareas: 
Tabla 8. Comparativa de tiempos de respuesta

\begin{tabular}{|c|c|c|c|c|}
\hline Ubicación & Tarea actual & $\begin{array}{l}\text { Tiempo } \\
\text { actual }\end{array}$ & Tareas SIGEST & $\begin{array}{l}\text { Tiempo } \\
\text { SIGEST }\end{array}$ \\
\hline Triaje & $\begin{array}{l}\text { Toma de datos del paciente: } \\
\text { datos completos, procedencia, } \\
\text { parentesco, sexo, edad, } \\
\text { etcétera. }\end{array}$ & $3 \mathrm{~min}$ & $\begin{array}{l}\text { Toma de datos del paciente; } \\
\text { ingresa el DNI se visualiza la } \\
\text { información del sistema. }\end{array}$ & 4 seg \\
\hline Triaje & $\begin{array}{l}\text { Buscar historial del paciente: } \\
\text { Solicitar a enfermera que busque } \\
\text { y traiga el historial del paciente. }\end{array}$ & $8 \mathrm{~min}$ & $\begin{array}{l}\text { Buscar historial del paciente: } \\
\text { Selecciona botón "Ver } \\
\text { historial", se visualizan cinco } \\
\text { últimas visitas. }\end{array}$ & $1 \mathrm{seg}$ \\
\hline Triaje & $\begin{array}{l}\text { Ingresar descripción del CIE } \\
\text { y código de CIE: Escribir el } \\
\text { diagnóstico y buscar en un PDF } \\
\text { el número de CIE relacionado. }\end{array}$ & $1 \mathrm{~min}$ & $\begin{array}{l}\text { Ingresar descripción del } \\
\text { CIE y código de CIE: Digitar } \\
\text { patología, muestra en forma } \\
\text { automática coincidencias y } \\
\text { CIE. }\end{array}$ & 5 seg \\
\hline Triaje & $\begin{array}{l}\text { Registrar diagnóstico, } \\
\text { seleccionar tópico y asignar } \\
\text { prioridad junto a la derivación: } \\
\text { Escribir en un ticket, según } \\
\text { color, el tópico a derivar y } \\
\text { el diagnóstico, y dárselo al } \\
\text { paciente. }\end{array}$ & $1 \mathrm{~min}$ & $\begin{array}{l}\text { Registrar diagnóstico, } \\
\text { seleccionar tópico y asignar } \\
\text { prioridad conjunto a la } \\
\text { derivación. Digitar diagnóstico } \\
\text { Se realiza automáticamente } \\
\text { dependiendo del tópico. }\end{array}$ & 30 seg \\
\hline Triaje & $\begin{array}{l}\text { Registrar atención: Indicar al } \\
\text { paciente el lugar donde dirigirse } \\
\text { y entregar la Hoja de Triaje. }\end{array}$ & $1 \mathrm{~min}$ & $\begin{array}{l}\text { Registrar atención: } \\
\text { Seleccionar el botón Crear y } \\
\text { enviar al paciente a Caja. }\end{array}$ & $1 \mathrm{seg}$ \\
\hline Caja & $\begin{array}{l}\text { Registrar al paciente: Solicitar } \\
\text { datos completos (procedencia, } \\
\text { parentesco, sexo, edad, etc.) }\end{array}$ & $3 \mathrm{~min}$ & $\begin{array}{l}\text { Registrar al paciente: Ingresar } \\
\text { DNl y visualizar data. }\end{array}$ & 4 seg \\
\hline Caja & $\begin{array}{l}\text { Generar recibo y finalizar } \\
\text { atención. }\end{array}$ & $1 \mathrm{~min}$ & $\begin{array}{l}\text { Registrar recibo y finalizar } \\
\text { atención. }\end{array}$ & 5 seg \\
\hline Admisión & Generar ficha y finalizar atención. & $1 \mathrm{~min}$ & $\begin{array}{l}\text { Registrar ficha y finalizar } \\
\text { atención. }\end{array}$ & 4 seg \\
\hline Admisión & Generar ficha y finalizar atención. & $1 \mathrm{~min}$ & Registrar ficha y finalizar. & 2 seg \\
\hline Tópico & $\begin{array}{l}\text { Registrar atención: Indicar al } \\
\text { paciente el lugar donde dirigirse } \\
\text { y entregar la Hoja de Triaje. }\end{array}$ & $3 \mathrm{~min}$ & $\begin{array}{l}\text { Registrar al paciente: Ingresar } \\
\text { DNl y visualizar data }\end{array}$ & 4 seg \\
\hline Tópico & $\begin{array}{l}\text { Registrar diagnóstico y finalizar } \\
\text { atención. }\end{array}$ & $2 \mathrm{~min}$ & $\begin{array}{l}\text { Registrar diagnóstico y } \\
\text { finalizar atención. }\end{array}$ & $1 \mathrm{~min}$ \\
\hline Tiempo total & & $27 \mathrm{~min}$ & Tiempo total & $2 \min$ \\
\hline
\end{tabular}

Elaboración propia 


\section{Resultados}

La solución se elaboró usando tecnologías Microsoft, debido a que el hospital cuenta con desarrollos en esta plataforma, lo que permitió la reutilización de los recursos; además, esta tecnología dispone de un conjunto de herramientas que dan una solución a los requerimientos no funcionales del usuario. Para el desarrollo se utilizó el patrón de software MVC (figura 14), con tres clases que se relacionan entre sí y distribuidos con sus componentes, tales como los beans, conexiones a base de datos, imágenes, librerías y vistas en el lenguaje HTML (HyperText Markup Language) para diseñar páginas web en las diferentes capas de la aplicación.

Figura 14. MVC del proyecto

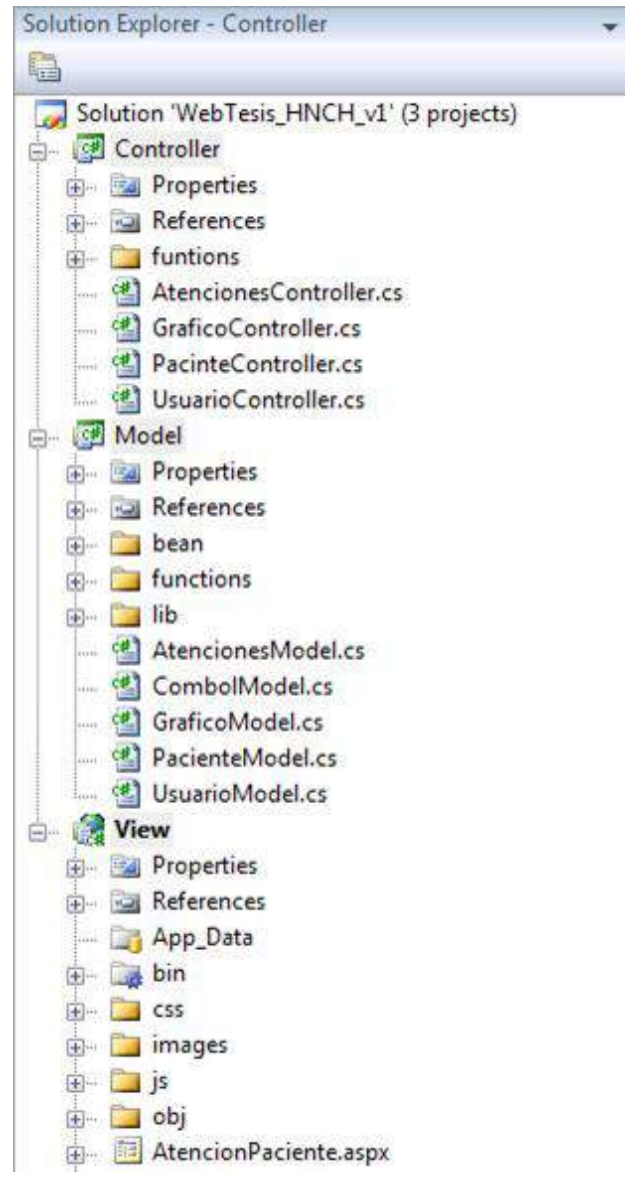

Elaboración propia 


\subsection{Ahorro de costos}

El sistema permitió reducir el uso del papel en el área de Emergencia, eliminándose la emisión del ticket de atención; de esa forma también se redujo el uso de tinta y mantenimiento de las impresoras. Además se acortaron los tiempos de atención, pues no se toman reiterativamente los datos y la información está consolidada. Si se tiene en cuenta la duración de una atención y su costo actual, considerando los datos de duración de atención obtenidos en verificación de sistema (ver tabla 8), se obtuvo lo siguiente:

\section{Tabla 9. Costos de personal de atención}

\begin{tabular}{|l|r|r|r|r|}
\hline \multicolumn{1}{|c|}{ Personal } & $\begin{array}{c}\text { Sueldo mensual } \\
\text { (S/) }\end{array}$ & $\begin{array}{c}\text { Costo por minuto } \\
\text { (S/) }\end{array}$ & Cantidad & $\begin{array}{c}\text { Costo por puesto } \\
\text { por minuto (S/) }\end{array}$ \\
\hline Enfermera IV & 1440,73 & 0,013340 & 2 & 0,0267 \\
\hline $\begin{array}{l}\text { Técnico en } \\
\text { enfermería II }\end{array}$ & 1091,00 & 0,010102 & 4 & 0,0404 \\
\hline
\end{tabular}

Elaboración propia

Tabla 10. Comparación de costos de atención

\begin{tabular}{|l|r|r|r|r|}
\hline \multicolumn{1}{|c|}{ Personal } & $\begin{array}{c}\text { Tiempo atención } \\
\text { actual (min) }\end{array}$ & $\begin{array}{r}\text { Costo actual } \\
\text { atención (S/) }\end{array}$ & $\begin{array}{c}\text { Tiempo atención } \\
\text { SIGEST (min) }\end{array}$ & $\begin{array}{c}\text { Costo } \\
\text { SIGEST (S/) }\end{array}$ \\
\hline Enfermera IV & 27 & 0,72036 & 2 & 0,05336 \\
\hline $\begin{array}{l}\text { Técnico en } \\
\text { enfermería II }\end{array}$ & 27 & 1,09102 & 2 & 0,08082 \\
\hline $\begin{array}{l}\text { Costo total por } \\
\text { una atención } \\
\text { (S/) }\end{array}$ & & 1,81138 & & 0,13418 \\
\hline $\begin{array}{l}\text { Costo total por } \\
250 \text { atenciones } \\
\text { (S/) }\end{array}$ & & 452,845 & & 33,545 \\
\hline
\end{tabular}

Elaboración propia

De la tabla 10 se desprende que por cada 250 atenciones realizadas se logra un ahorro de S/ 419,3. 


\subsection{Beneficios intangibles}

En relación a los beneficios intangibles, se han valorado los siguientes aspectos:

i. Enfoque de gestión por procesos, mediante la trazabilidad de las actividades involucradas y el control de tiempos, facilitando la gestión de indicadores.

ii. Control y seguimiento de pacientes atendidos por el área de Emergencia.

iii. Acceso a la información histórica de los pacientes y sus atenciones.

iv. Aprovechamiento de recursos tecnológicos instalados y de licencias de software informático que posee el hospital.

v. Integración de la unidad de Triaje con las diferentes unidades involucradas durante el proceso de atención.

vi. Capacidad para generar datos estadísticos y reportes requeridos para verificar el impacto que genera el sistema web.

vii. Mejora de la calidad del servicio a los pacientes, porque la búsqueda rápida de patologías permite la identificación del nivel de prioridad del paciente.

viii. Disminución del tiempo de atención a los pacientes.

\section{Conclusiones y recomendaciones}

\subsection{Conclusiones}

i. A partir del modelo AS-IS se detectaron actividades redundantes, actividades con información no integrada o no consolidada y cuellos de botella.

ii. La automatización del modelo TO-BE brindó una mejora de 25 minutos en el tiempo de atención con respecto al modelo base.

iii. Se estandarizó la información de la base de datos para integrar la información requerida por cada participante en el proceso de atención en Emergencia.

iv. Los resultados favorables de las pruebas unitarias de los métodos principales permitieron la aprobación por parte del hospital. 


\subsection{Recomendaciones}

i. Usar los patrones MVC para próximos desarrollos, pues estos facilitan la reutilización de código.

ii. Considerar la redundancia en los servidores, switches y enlaces a nivel de hardware, así como de base de datos a nivel de software, para mantener disponible el sistema web y asegurar la continuidad de las operaciones.

iii. Continuar con la automatización de otros procesos interrelacionados para ampliar el alcance del proceso y ampliar sus beneficios.

iv. Considerar el estándar HL7 para el manejo e intercambio de información.

\section{Referencias}

Arias, R. y Fisher, B. (2013). A Qualitative Methodology for the Design of Visual Analytic Tools for Emergency Operation Centers. 46th Hawail International Conference on System Sciences, (pp.126-135). California. DOl:10.1109/HICSS. 2013.57

Ma, W., Goldstein, B., Ma, Y., Sun, D., y Burgess, S. (2012). MedlinePlus Mobile: Consumer Health Information On-the-Go. IT Professional, 14(3), 44-49. DOl: 10.1109/MITP.2012.20

Microsoft (2007). Información general sobre ASP.NET. Recuperado de https://msdn. microsoft. com/es-es/library/4w3ex9c2(v=vs.100).aspx

Mizumoto, T., Imazu, S., Sun, W. Shibata, N., y Yasumoto, K. (2012). Emergency Medical Support System for Visualizing Locations and Vital signs of Patients in Mass Casualty Incidents. 2012 IEEE International Conference on Pervasive Computing and Communications Workshops, (pp.740-745). DOl:10.1109/PerComW.2012. 6197611

Mowbray, T. y Lilly, S. (1999). Books. IBM Systems Journal, 38(1), 122-124. DOl:10.1147/ sj.381.0122

Osterwalder, A., Pigneur, y Clark, T. (2010). Business Model Generation: A Handbook for Visionaries, Game Changers, and Challengers. Nueva Jersey: John Wiley \& Sons.

Padmanabhan, N., Burstein, F., Churilov, L., Wassertheil, J., Hornblower, B., y Parker, N. (2006). A Mobile Emergency Triage Decision Support System Evaluation. 39th Annual Hawaii International Conference on System Sciences (HICSS'06), 5, 96b. IEEE. DOl:10.1109/ HICSS.2006.17

Ministerio de Salud (2006). Norma técnica de salud para la atención integral de salud. Lima, Perú. 
Congreso de la República del Perú (Diciembre 19, 2001). Reglamento de la Ley N. ${ }^{\circ} 27604$, que modifica la Ley General de Salud N. ${ }^{\circ}$ 26842, respecto de la obligación de los establecimientos de salud a dar atención médica en caso de emergencias y partos.

Sakanushi, K., Hieda, T., Shiraishi, T., Ode, Y., Takeuchi, Y., Imai, M., ... Tanaka, H. (2011). Electronic Triage System: Casualties Monitoring System in the Disaster Scene. 2011 International Conference on P2P, Parallel, Grid, Cloud and Internet Computing, (pp. 317322). IEEE. DOl:10.1109/3PGCIC.2011.58

Selfa, D., Carrillo, M., y Boone, D. (2006). A Database and Web Application based on MVC Architecture. 16th International Conference on Electronics, Communications and Computers (CONIELECOMP'O6), (p.48). IEEE. DOI:10.1109/ CONIELECOMP.2006.6

Simmons, E. (2004). Requirements Triage: What Can We Learn from a "Medical" Approach? IEEE Software, 21(4), 86-88. DOl:10.1109/MS.2004.25

Torres, F. (2009). Integración del PMBOK al RUP para proyectos de desarrollo de software. Octava Conferencia Iberoamericana en Sistemas, Cibernética e Informática: ClSCI 2009. Recuperado de http://www.iiis.org/CDs2008/ CD2009CSC/ClSCl2009/PapersPdf/ C690Ml.Pdf

Yeh, S.-C., Hwang, W.-Y., Huang, T.-C., y Liu, W.-K. (2011). U-Healthcare System Deployment. 2011 IEEE 11th International Conference on Bioinformatics and Bioengineering, (pp. 137142). IEEE. DOl:10.1109/BIBE.2011.30

\section{Glosario}

- Emergencia: Se debe atender al paciente, quien corre el riesgo de perder su vida.

- Urgencia: Una situación en la que el paciente no corre el riesgo de perder su vida, pero debe ser atendido con rapidez.

- Canvas: Estructura sintetizada del modelo de negocio de una empresa.

- Host: Componente terminal físico conectado a una red.

- Switch: Dispositivo lógico de interconexión de redes. 


\section{Anexos}

Figura 15. Solicitud de nueva atención

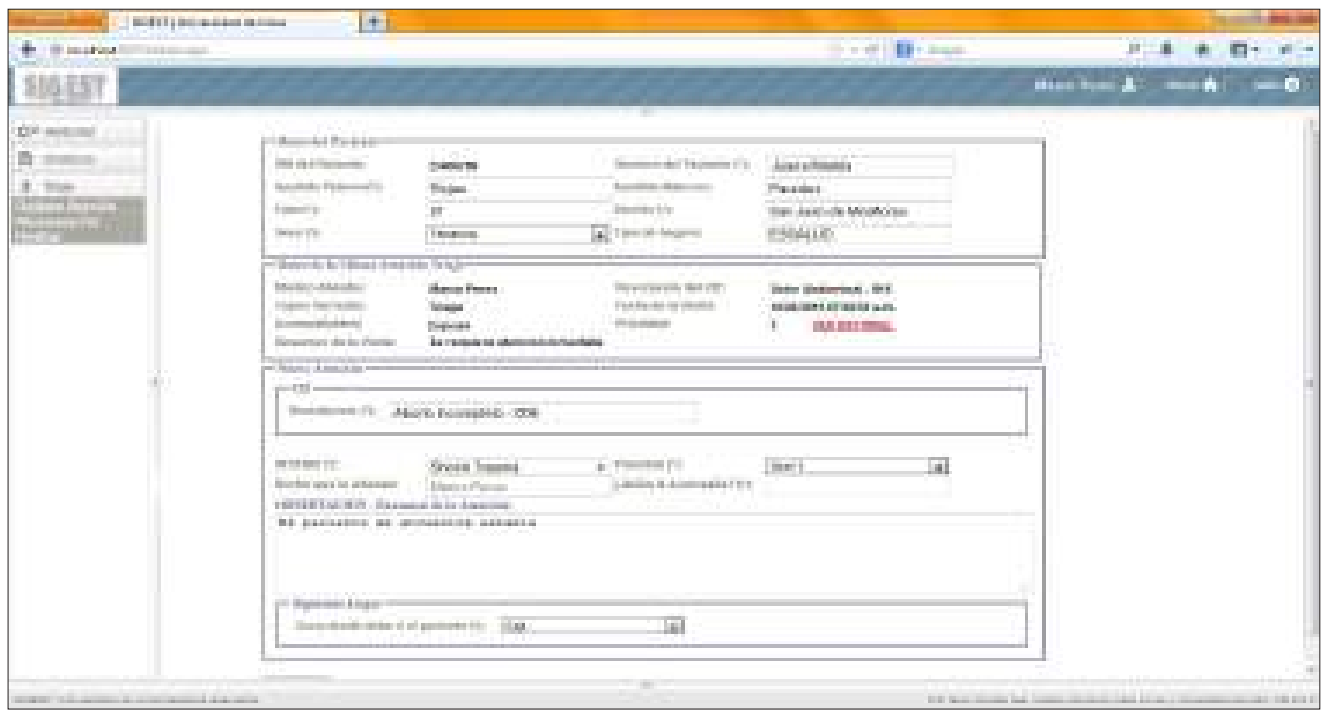

Elaboración propia

Figura 16. Interfaz de paciente nuevo

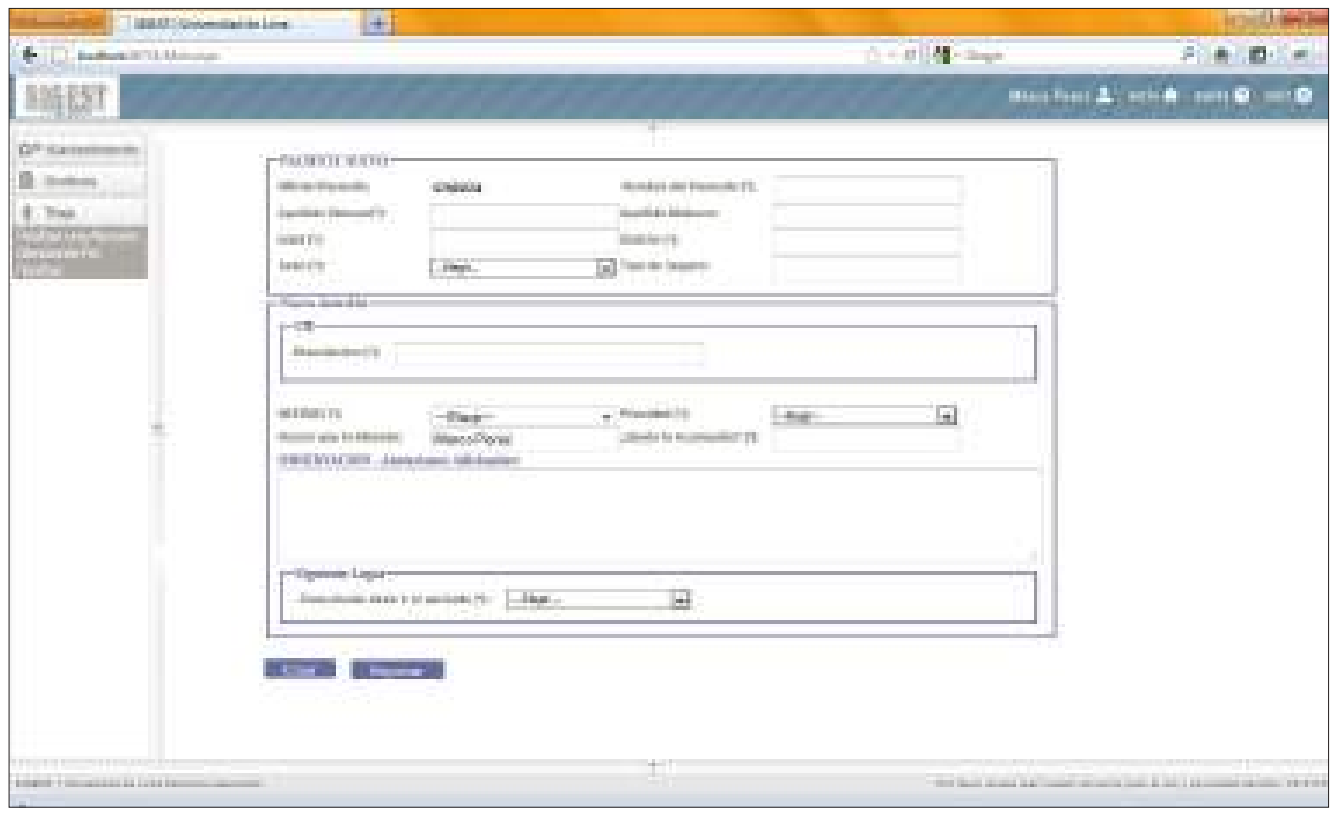

Elaboración propia 